\title{
INVESTIGATING CONTRIBUTIONS OF GASES, METEOROLOGICAL PARAMETERS, AND AEROSOLS TOWARDS TROPOSPHERIC OZONE VARIABILITIES OVER MEGACITY LAHORE (PAKISTAN)
}

\author{
PARveZ, S. - RANA, A. D. - Ul-HaQ, Z. - BAtool, S. A. - Ali, M. - TARIQ, S. - \\ MAHMOOD, K. - BANO, S. \\ GIS and Remote Sensing Group, Department of Space Science, University of the Punjab \\ Lahore, Pakistan \\ ${ }^{*}$ Corresponding author \\ e-mail:zia.spsc@yahoo.com
}

(Received $19^{\text {th }}$ Mar 2019; accepted $4^{\text {th }}$ Jul 2019)

\begin{abstract}
We investigated contributions of some tropospheric gases, meteorological parameters, and aerosol burden measured as aerosol optical depth (AOD) towards Tropospheric Ozone $\left(\mathrm{TropoO}_{3}\right)$ variabilities over Lahore by developing a stepwise multiple linear regression model. The tropospheric gases include; Methane $\left(\mathrm{CH}_{4}\right)$, Carbon Monoxide $(\mathrm{CO})$, Carbon Dioxide $\left(\mathrm{CO}_{2}\right)$, and Sulphur Dioxide $\left(\mathrm{SO}_{2}\right)$, whereas the meteorological parameters include; cloud fraction (CLF), outgoing long-wave radiation (OLR), relative humidity $(\mathrm{RH})$, temperature (Temp), and surface wind-speed (WS). Data products of these parameters have been retrieved from various satellite sensors during October 2004 -September 2015. Time series distributions of both observed and modeled $\mathrm{TropoO}_{3}$ concentrations show similar increasing trends except for few months. Moreover, monthly-means of these observed and modeled $\mathrm{TropoO}_{3}$ concentrations indicate similar decreasing trends with maximum during spring and summer (monsoon) seasons. Based upon weighted contributions of standardized regression coefficients, modeled $\mathrm{TropoO}_{3}$ concentration was found dependent upon OLR[0.458], WS[0.262], CLF[0.256] TropoSO $2[-0.097]$, TropoCO[-0.231], and $\mathrm{RH}[-0.320]$ out of all parameters used in this study. Modeled $\mathrm{TropoO}_{3}$ concentrations were validated against an independent source of $\mathrm{TropoO}_{3}$ concentrations retrieved from original subtracted product of OMI-MLS over Lahore and the results were found in good agreement. The study is unique and would be helpful for further research investigations.
\end{abstract}

Keywords: regression modeling, atmospheric outlook of Lahore, ozone contributors, ecology, atmospheric remote sensing

\section{Introduction}

$\mathrm{TropoO}_{3}$ is one of the short-lived but major atmospheric pollutants that plays a key role towards climate change (UNEP and WMO, 2011) by modifying the chemical composition of the troposphere and its oxidation capacity (Gauss et al., 2003). It is considered as a primary precursor for the production of hydroxyl radical $(\mathrm{OH})$, that acts as an oxidizing agent severely disturbing the occurrence of tropospheric trace gases (Seinfeld and Pandis, 2016). TropoO $_{3}$ absorbs short-wave solar radiations and after attenuation re-radiates it in the form of long-wave radiations (Shan et al., 2008; Pal, 2010; Tan et al., 2014). TropoO 3 has harmful effects on human health, vegetation health $\&$ yield, and sensitive ecosystems (EPA, 2003; The Royal Society, 2008; Avnery et al., 2011; Burney and Ramanathan, 2014). Also, $\mathrm{TropoO}_{3}$ is one of the major anthropogenic greenhouse gases, which is responsible for global warming (IPCC, 2013). Ozone in troposphere is either produced from chemical reactions of $\mathrm{NO}_{\mathrm{x}}, \mathrm{CH}_{4}, \mathrm{CO}$, and volatile organic compounds (VOCs) through non-linear, complex, and feedback-regulated processes in the presence of solar radiations (El-Fadel et al., 2002; Pulikesi et al., 2006; 
Stevenson et al., 2006; Young, 2013; Seinfeld and Pandis, 2016) or transported directly from the stratosphere (Hsu and Prather, 2009). From an ecological point of view, both increased emission and destruction of VOCs by $\mathrm{TropoO}_{3}$ may affect several of their multiple functions (Lerdau and Slobodkin, 2002; Holopainen, 2004; Van, 2009; Yuan et al., 2009). There are a number of anthropogenic and natural sources of these $\mathrm{TropoO}_{3}$ precursors e.g.; crop-residue burning, biomass burning, fossil fuel combustion, enhancement of volatile organic compounds through convections of $\mathrm{CO}$ and aerosols, vehicular emissions, industrial processing, energy production activities, waste management, lightning $\mathrm{NO}_{\mathrm{x}}$, and soil emissions (Jaeglé et al., 2004; Satheesh and Moorthy, 2005; Stevenson et al., 2006; Aghedo et al., 2007; Wang et al., 2012; Ganesan et al., 2013; Ul-Haq et al., 2014). Anthropogenic activities pose numerous threats to the functioning, structural growth and diversity of natural and semi-natural ecosystems (Bobbink, 1989). Globally, an increase of $36 \%$ in TropoO 3 burden has been reported since 1850 (Cooper et al., 2014). TropoO ${ }_{3}$ has strong effects on ecological interactions based on VOC signaling (Pinto et al., 2010).

Various natural, meteorological and anthropogenic factors are responsible for $\mathrm{TropoO}_{3}$ concentration variability. Natural factors include; seasons, Earth-Sun distance, and location (altitude, latitude, and longitude), meteorological factors include; temperature, precipitation, humidity, soar flux, and wind speed, while anthropogenic factors include; emissions from precursor pollutants, biomass burning, vehicular emissions, and industrial processing (Ahmad and Aziz, 2013; Iqbal et al., 2014). Aerosols decrease TropoO$_{3}$ formation in polluted areas (Satheesh and Moorthy, 2005; Xing et al., 2017) and also decline its concentration after reacting with tropospheric gases (Sahoo et al., 2005). The primary objective of this study is to investigate the potential contributions of major tropospheric gases, meteorological parameters, and aerosol burden towards $\mathrm{TropoO}_{3}$ variability over Lahore. TropoO ${ }_{3}$ monitoring is being carried out either by onboard satellite sensors (Cooper et al., 2014; Ziemke et al., 2014) or via ground-based stations (Fioletov et al., 2008). Remote sensing techniques provide cost-effective, synoptic and repetitive coverages for spatio-temporal monitoring of tropospheric gases, meteorological parameters, and aerosol burden either at large or small scales. Whereas, data acquisition via ground-based stations is expensive, time-consuming, and almost impossible for inaccessible areas. The present study is carried out by retrieving datasets from onboard satellite sensors including; AIRS, AMSU-A, MODIS, MOPITT, OMI, and TRMM, as well as GLDAS model. To the best of our knowledge, no detailed study has so far been published for assessing $\mathrm{TropoO}_{3}$ variability as functions of remotely sensed major tropospheric gases, meteorological parameters, and aerosol burden over Lahore.

\section{Material and Methods}

\section{Location and description of the study area}

Metropolitan corporation Lahore $\left(31.5204^{\circ} \mathrm{N}, 74.3587^{\circ} \mathrm{E}\right)$ is the capital city of the Punjab province in Pakistan. As per $6^{\text {th }}$ Population Census 2017 results, Lahore is the second-most populous city and financial hub of Pakistan after Karachi having population over 11 million and an area of $1,172 \mathrm{~km}^{2}$ (Figure 1). Lahore is bounded by Sheikhupura District on the north and west bifurcated by Ravi River, Kasur District on its south, and Wagah Border along Indian province Punjab on the east. Lahore is the $16^{\text {th }}$ largest and rapidly growing city in the world (CMF, 2018). Being financial hub, Lahore contains all types of Industrial, manufacturing, construction, business, and IT related activities 
including but not limited to; pharmaceutical and chemical factories, steel mills, construction materials, power generation, manufacturing of motor vehicles, home appliance, electronics, leather goods, and paper products (Alam et al., 2012; Ali et al., 2014). Major air pollution sources in Lahore include automobile emissions, road dust, and biomass burning along with air pollutants transported from nearby regions (Alam et al., 2010, 2014). The Indian states of Punjab, Haryana, and Uttar Pradesh (shown in Figure 1) are reported to be the well-known air-pollutant emission sources from large scale rice and wheat crops generated residue burning and periodically transported to Lahore (Sidhu and Beri, 2008; Badarinath et al., 2009; Mishra and Shibata, 2012). Lahore falls under hot semi-arid and wet category based on the Köppen climate classification system with highly hot and wet summers (heavy monsoon rainfalls with frequent dust storms during June to September), while dry and cold winters (from December to February). Spring (March to May) and autumn (September to November with extensive crop residue burning in the surrounding regions) seasons are also observed in Lahore (Ali et al., 2014).

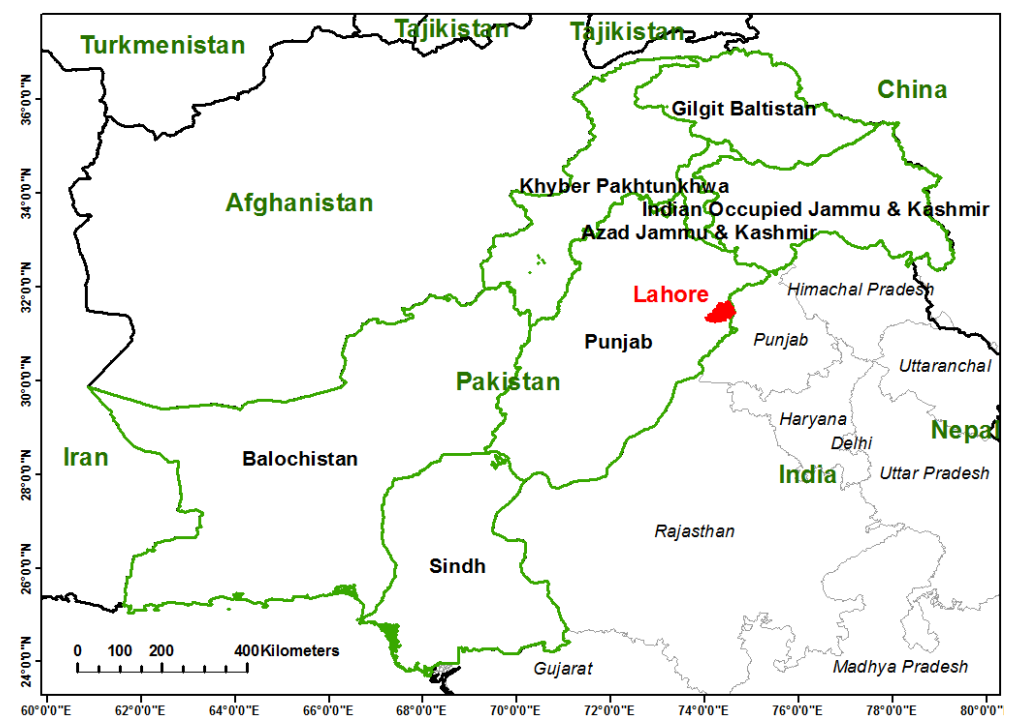

Figure 1. Map showing the geographical location of Lahore $\left(31.5204^{\circ} \mathrm{N}, 74.3587^{\circ} \mathrm{E}\right)$.

(Source: https://www.statsilk.com/maps/download-free-shapefile-maps)

\section{Datasets}

Host of satellite borne sensors have been providing online data products for monitoring and analyzing atmospheric gases, meteorological parameters, and aerosol burden measured as aerosol optical depth (AOD). For current study, we retrieved monthly-mean data products of TropoCH $\mathrm{CH}_{4}$, TropoCO $\mathrm{CO}_{2}, \mathrm{CLF}$, and OLR, while daily datasets of $\mathrm{RH}$, and surface temperature over Lahore from Atmospheric Infrared Sounder (AIRS, Pagano et al., 2003; Abed, 2017) and the Advanced Microwave Sounding Unit-A (AMSU-A, Aumann et al., 2003) onboard AURA satellite operational since 2002. The combined use of AIRS and AMSU-A data products basically offers new as well as improved measurements (Susskind et al., 2003). Data products of TropoNO $\mathrm{N}_{2}$ (daily), $\mathrm{TropoO}_{3}$ (monthly-mean), and planetary boundary layer $\mathrm{SO}_{2}$ (monthly-mean) were acquired from NASA's EOS Ozone Monitoring Instrument (OMI) onboard Aura satellite launched in a sun-synchronous polar orbit on July 15, 2004 at an altitude of $705 \mathrm{~km}$ and inclination of 
98.2 ${ }^{\circ}$ (Levelt et al., 2006a; Ziemke et al., 2011). OMI provides almost global coverage daily with a resolution of $13 \times 24 \mathrm{~km}^{2}$ at nadir while $13 \times 100 \mathrm{~km}^{2}$ at off-nadir (Boersma et al., 2006). It consists of hyperspectral imaging sensors to record radiances in the range of 270-500 nm (Levelt et al., 2006a,b). Additionally, monthly-mean data of TropoCO was retrieved from MOPITT onboard Terra, whereas, AOD from MODIS onboard Aqua deep blue. The datasets of surface level precipitation rate and near surface wind speed over Lahore were obtained from Tropical Rainfall Measuring Mission (TRMM, Liu et al., 2012) and Global Land Data Assimilation System (GLDAS, Fang et al., 2009), respectively. For validation of our resultant modeled, the original $\mathrm{TropoO}_{3}$ data product of subtracted monthly-means of Total Column Ozone retrievable from OMI and Stratospheric Column Ozone retrievable from MSL onboard Aura satellite was utilized (Ziemke et al., 2006). Details about all the data products of major tropospheric gases, meteorological parameters, and aerosols used for this study are given in Table 1.

\section{Methodology}

We developed a bivariate correlation matrix representing tropospheric gases $\left(\mathrm{CH}_{4}\right.$, $\mathrm{CO}, \mathrm{CO}_{2}, \mathrm{NO}_{2}, \mathrm{O}_{3}$, and $\mathrm{SO}_{2}$ ), meteorological parameters (CLF, OLR, PR, RH, Temp, and WS), and aerosol burden over Lahore using monthly-mean remotely sensed data during October 2004 - September 2015 (shown in Table 2). Each parameter mentioned in Table 2 has either positive value (shown in blue colored bar) or negative value (shown in red colored bar), which indicates the type of correlation between the respective parameters. Longer the length of colored bars (either blue or red), stronger would be the correlation (either positive or inverse, respectively) between the corresponding parameters. Whereas, colored bars having smaller lengths (smaller values either positive or negative) indicate weaker correlations. In Table 2, correlation values written in bold text are significant at the 0.01 level (2-tailed), whereas, in italic text are significant at the 0.05 level (2-tailed). Respective statistical significance values, against each correlations of Table 2, are mentioned in Table 3. The highest statistical significance values at the 0.01 level (2-tailed) are highlighted in bold text, whereas, the statistical significance values at the 0.05 level (2-tailed) are mentioned in italic text in Table 3. The values written in regular text indicate least significance values. The highest correlation value [0.73] with 99\% significance at 0.00 level (2-tailed) indicates that PR \& CLF (meteorological parameters) are found to be positively highly correlated with each other in the study area during the observation period. Other higher positive correlations significant at 0.00 level (2-tailed) are; RH \& CLF [0.70], RH \& PR [0.69], Temp \& AOD [0.69], and Temp \& OLR [0.66]. Next equally higher but opposite correlations significant at 0.00 level (2tailed) are; OLR \& $\mathrm{TropoO}_{3}$ [0.61], and $\mathrm{TropoO}_{3} \&$ TropoCO [-0.61]. Remaining relatively higher correlations significant at 0.00 level (2-tailed) are; WS \& $\mathrm{TropoO}_{3}$ [0.57], $\mathrm{TropoCO}_{2} \& \mathrm{TropoCH}_{4}$ [0.54], $\mathrm{RH} \& \mathrm{TropoCH}_{4}$ [0.54], WS \& TropoCO [-0.54], and RH \& OLR [-0.52]. The parameters used in this study having weaker correlations with each other significant at 0.00 level (2-tailed) are (in descending order); OLR \& CLF [-0.48], RH \& $\mathrm{TropoO}_{3}$ [-0.46], PR \& AOD [0.45], PR \& $\mathrm{TropoCH}_{4}$ [0.45], RH \& AOD [0.41], WS \& $\mathrm{TropoCO}_{2}$ [0.40], Temp \& $\mathrm{TropoCH}_{4}$ [0.39], AOD \& $\mathrm{TropoCH}_{4}$ [0.34], Temp \& PR [0.33], OLR \& TropoCO [-0.31], Temp \& $\mathrm{TropoO}_{3}$ [0.28], $\mathrm{TropoNO}_{2} \&$ $\mathrm{TropoCO}_{2}$ [0.28], CLF \& $\mathrm{TropoCH}_{4}$ [0.27], WS \& CLF [0.25], TropoCO 2 \& TropoCO $[-0.23]$, WS \& $\mathrm{TropoNO}_{2}[0.23]$, and $\mathrm{TropoSO}_{2} \& \mathrm{TropoO}_{3}[-0.23]$. Out of all the tropospheric gases used in this study, $\mathrm{TropoO}_{3}$ and TropoCO are found to be highly but inversely correlated with each other, whereas, $\mathrm{TropoCO}_{2}$ and $\mathrm{TropoCH}_{4}$ are next highly 


$$
-13537 \text { - }
$$

and positively correlated gases significant at 0.01 level (2-tailed). Other tropospheric gases with weaker correlations including positive as well as inverse significant at 0.00 level (2-tailed) are; $\mathrm{TropoNO}_{2} \& \mathrm{TropoCO}_{2}$ [0.28], $\mathrm{TropoCO}_{2} \& \mathrm{TropoCO}[-0.23]$, and $\mathrm{TropoSO}_{2} \& \mathrm{TropoO}_{3}[-0.23]$. Only one correlation (least and positive) significant at 0.05 level (2-tailed) was found between $\mathrm{TropoSO}_{2} \& \mathrm{TropoNO}_{2}$ [0.18]. Positive as well as inverse correlations with $95 \%$ significance at 0.05 level (2-tailed) observed from this study are; OLR \& AOD [0.22], CLF \& $\mathrm{TropoSO}_{2}$ [-0.22], AOD \& TropoCO [-0.21], CLF \& AOD [0.19], WS \& RH [-0.19], and $\mathrm{TropoSO}_{2} \& \mathrm{TropoNO}_{2}$ [0.18]. All these correlations are also comparatively weaker ones.

Table 1. Metadata of data retrieved from different satellite sensors (level-3 products) and models used in this study

\begin{tabular}{|c|c|c|c|c|c|c|}
\hline Product Name/Version & $\begin{array}{c}\text { Sensor/ } \\
\text { Model }\end{array}$ & Units & $\begin{array}{c}\text { Spatial } \\
\text { Resolution }\end{array}$ & \begin{tabular}{|r|} 
Temporal \\
Resolution
\end{tabular} & $\begin{array}{c}\begin{array}{c}\text { Observation } \\
\text { Period }\end{array} \\
\end{array}$ & Description \\
\hline \multicolumn{7}{|l|}{ a) Tropospheric Gases } \\
\hline $\begin{array}{l}\text { TropoCH4 - Tropospheric Methane } \\
\text { (AIR3STM_VMR_A.700hPa v006) }\end{array}$ & $\begin{array}{c}\text { AIRS/ } \\
\text { AMSU- } \\
\text { A }\end{array}$ & ppbv & $0.25^{\circ} \times 0.25^{\circ}$ & Daily & $\begin{array}{l}2002- \\
\text { current }\end{array}$ & Tropospheric \\
\hline $\begin{array}{c}\text { TropoCO - Tropospheric Carbon } \\
\text { Monoxide } \\
\text { (MOP03JM v007) }\end{array}$ & MOPITT & ppbv & $1^{\circ} \times 1^{o}$ & Daily & $\begin{array}{l}2000- \\
\text { current }\end{array}$ & Tropospheric \\
\hline $\begin{array}{c}\text { TropoCO }_{2} \text { - Tropospheric Carbon } \\
\text { Dioxide } \\
\text { (AIR3C2M mole fraction v005) } \\
\end{array}$ & $\begin{array}{l}\text { AIRS/ } \\
\text { AMSU- } \\
\text { A }\end{array}$ & ppm & $1^{\circ} \times 1^{\circ}$ & Daily & $\begin{array}{l}2002- \\
\text { current }\end{array}$ & Tropospheric \\
\hline $\begin{array}{c}\text { TropoNO }_{2}-\text { Tropospheric Nitrogen } \\
\text { Dioxide } \\
(\mathrm{OMNO} 2 \mathrm{~d} \text { v003) }\end{array}$ & OMI & $\begin{array}{c}\mathrm{x} 10^{15} \\
\text { molecules } \\
\mathrm{cm}^{-2}\end{array}$ & $0.25^{\circ} \mathrm{x} 0.25^{\circ}$ & Daily & $\begin{array}{l}2004- \\
\text { current }\end{array}$ & Tropospheric \\
\hline $\begin{array}{c}\text { TropoO }_{3} \text { - Tropospheric Ozone } \\
\text { (AIR3STM_VMR_A.700hPa v006) }\end{array}$ & $\begin{array}{c}\text { AIRS/ } \\
\text { AMSU- } \\
\text { A } \\
\end{array}$ & ppbv & $0.25^{\circ} \times 0.25^{\circ}$ & Daily & $\begin{array}{l}2002- \\
\text { current }\end{array}$ & Tropospheric \\
\hline $\begin{array}{c}\text { TropoSO }_{2}-\text { Tropospheric Sulphur } \\
\text { Dioxide } \\
\text { (OMSO2e_PBL v003) }\end{array}$ & OMI & DU & $0.25^{\circ} \times 0.25^{\circ}$ & Daily & $\begin{array}{l}2004- \\
\text { current }\end{array}$ & $\begin{array}{l}\text { Planetary } \\
\text { boundary } \\
\text { layer }\end{array}$ \\
\hline \multicolumn{7}{|l|}{ b) Meteorological Parameters } \\
\hline $\begin{array}{l}\text { CLF - Cloud Fraction } \\
\text { (AIRX3STM v006) }\end{array}$ & $\begin{array}{c}\text { AIRS/ } \\
\text { AMSU- } \\
\text { A }\end{array}$ & Unit less & $1^{\circ} \times 1^{\circ}$ & Daily & $\begin{array}{l}2002- \\
\text { current }\end{array}$ & $\begin{array}{l}\text { Total } \\
\text { column }\end{array}$ \\
\hline $\begin{array}{c}\text { OLR - Outgoing Long-wave } \\
\text { Radiation } \\
\text { (AIRX3STM v006) } \\
\end{array}$ & $\begin{array}{c}\text { AIRS/ } \\
\text { AMSU- } \\
\text { A }\end{array}$ & $\mathrm{W} \mathrm{m}^{-2}$ & $1^{\circ} \times 1^{\circ}$ & Daily & $\begin{array}{l}2002- \\
\text { current }\end{array}$ & $\begin{array}{l}\text { Top of the } \\
\text { atmosphere }\end{array}$ \\
\hline $\begin{array}{c}\text { PR }- \text { Precipitation Rate } \\
\text { (TMPA/3B43 v007) }\end{array}$ & TRMM & $\mathrm{mm} \mathrm{month}^{-1}$ & $0.25^{\circ} \mathrm{x} 0.25^{\circ}$ & Monthly & $\begin{array}{l}1998- \\
\text { current }\end{array}$ & Surface level \\
\hline $\begin{array}{l}\text { RH - Relative Humidity } \\
\quad \text { (AIRX3STD v006) }\end{array}$ & $\begin{array}{c}\text { AIRS/ } \\
\text { AMSU- } \\
\text { A }\end{array}$ & $\%$ & $1^{\circ} \times 1^{\circ}$ & Daily & $\begin{array}{l}2002- \\
\text { current }\end{array}$ & $925 \mathrm{hPa}$ \\
\hline $\begin{array}{c}\text { Temp - Air Temperature } \\
\text { (AIRX3STD v006) }\end{array}$ & $\begin{array}{c}\text { AIRS/ } \\
\text { AMSU- } \\
\text { A } \\
\end{array}$ & Kelvin & $1^{\circ} \times 1^{\circ}$ & Daily & $\begin{array}{l}2002- \\
\text { current }\end{array}$ & $925 \mathrm{hPa}$ \\
\hline $\begin{array}{c}\text { WS - Wind Speed } \\
(\text { GLDAS_NOAH025_M v2.1) }\end{array}$ & $\begin{array}{c}\text { GLDAS } \\
\text { Model } \\
\end{array}$ & $\mathrm{m} \mathrm{s}^{-1}$ & $0.25^{\circ} \times 0.25^{\circ}$ & Monthly & $\begin{array}{l}2000- \\
\text { current }\end{array}$ & $\begin{array}{c}\text { Near surface } \\
\text { level }\end{array}$ \\
\hline \multicolumn{7}{|l|}{ c) Aerosols } \\
\hline $\begin{array}{c}\text { AOD - Aerosol burden measured as } \\
\text { Aerosol Optical Depth } \\
\text { (MOD08_M3_550 v006) }\end{array}$ & MODIS & Unit less & $1^{\circ} \times 1^{\circ}$ & 1-2 days & $\begin{array}{l}2002- \\
\text { current }\end{array}$ & $\begin{array}{l}\text { Total } \\
\text { column }\end{array}$ \\
\hline \multicolumn{7}{|l|}{$\begin{array}{l}\text { d) Independent data product of } \\
\text { TropoO3 for Model Validation }\end{array}$} \\
\hline $\begin{array}{c}\text { TropoO }_{3}-\text { Tropospheric Ozone } \\
\text { OMI/MLS tropospheric ozone (original } \\
\text { product) }\end{array}$ & $\begin{array}{l}\text { OMI- } \\
\text { MLS }\end{array}$ & ppbv & $1^{\circ} \times 1.25^{\circ}$ & $\begin{array}{l}\text { Monthly- } \\
\text { mean }\end{array}$ & $\begin{array}{l}2004- \\
\text { current }\end{array}$ & Tropospheric \\
\hline
\end{tabular}




$$
-13538 \text { - }
$$

Table 2. Bivariate correlation matrix representing monthly-mean data of tropospheric gases including TropoCH $\mathrm{CH}_{4}(\mathrm{ppbv})$, TropoCO (ppbv), TropoCO 2 (ppm), TropoNO $\mathrm{O}_{2}\left(x 10^{15}\right.$ molecules $\left.\mathrm{cm}^{-2}\right)$ and TropoSO $\mathrm{S}_{2}(\mathrm{DU})$; major meteorological parameters including cloud fraction (CLF, unit less), outgoing long-wave radiation $\left(O L R, W m^{-2}\right)$, precipitation rate $\left(P R, \mathrm{~mm} \mathrm{month}^{-1}\right)$, relative humidity $(\mathrm{RH}, \%)$, temperature at $925 \mathrm{hPa}$ (Temp, K) and surface wind speed (WS, $m$ $s^{-1}$ ); and aerosol burden measured as aerosol optical depth (AOD, unit less) over Lahore during October 2004 - September 2015

\begin{tabular}{|c|c|c|c|c|c|c|c|c|c|c|c|c|}
\hline Parameters & TropoCH $_{4}$ & TropoCO & $\mathrm{TropoCO}_{2}$ & $\mathrm{TropoNO}_{2}$ & $\mathrm{TropoO}_{3}$ & TropoSO $_{2}$ & AOD & CLF & OLR & PR & RH & Temp \\
\hline TropoCO & -0.14 & & & & & & & & & & & \\
\hline $\mathrm{TropoCO}_{2}$ & 0.54 & -0.23 & & & & & & & & & & \\
\hline $\mathrm{TropoNO}_{2}$ & -0.15 & 0.01 & 0.28 & & & & & & & & & \\
\hline $\mathrm{TropoO}_{3}$ & -0.07 & -0.61 & 0.14 & -0.06 & & & & & & & & \\
\hline TropoSO $_{2}$ & -0.02 & 0.13 & 0.08 & 0.18 & -0.23 & & & & & & & \\
\hline AOD & 0.34 & 0.21 & -0.01 & 0.07 & 0.09 & 0.01 & & & & & & \\
\hline CLF & 0.27 & -0.17 & 0.01 & -0.09 & -0.07 & 0.22 & 0.19 & & & & & \\
\hline OLR & 0.07 & -0.31 & -0.06 & -0.15 & 0.61 & -0.05 & 0.22 & -0.48 & & & & \\
\hline PR & 0.45 & -0.16 & 0.02 & -0.15 & 0.00 & -0.12 & 0.45 & $\begin{array}{l}0.73 \\
\end{array}$ & -0.10 & & & \\
\hline RH & 0.54 & 0.12 & 0.03 & -0.15 & -0.46 & -0.03 & 0.41 & 0.70 & -0.52 & 0.69 & & \\
\hline Temp & 0.39 & -0.16 & -0.11 & -0.14 & 0.28 & 0.01 & 0.69 & -0.09 & 0.66 & 0.33 & 0.11 & \\
\hline WS & -0.12 & -0.54 & 0.40 & 0.23 & 0.57 & -0.13 & 0.04 & 0.25 & 0.10 & 0.14 & 0.19 & -0.11 \\
\hline
\end{tabular}

Correlations in bold text are significant at the 0.01 level (2-tailed). Correlations in Italic are significant at the 0.05 level (2-tailed). Blue color indicates positive correlations, and red color indicates negative/inverse correlations. Longer the length of colored bars (either blue or red), stronger would be the correlation (either positive or inverse, respectively) between the corresponding parameters

Table 3. Based upon correlation matrix discussed in Table 2 above, matrix showing statistical significance values of tropospheric gases including TropoCH $\mathrm{TH}_{4}$ (ppb), TropoCO (ppbv), TropoCO $\mathrm{CO}_{2}$ (ppm), TropoNO $\mathrm{N}_{2}\left(\times 10^{15}\right.$ molecules $\left.\mathrm{cm}^{-2}\right)$ and TropoSO $\mathrm{S}_{2}(\mathrm{DU}) ;$ major meteorological parameters including cloud fraction (CLF, unit less), outgoing long-wave radiation $\left(O L R, W \mathrm{~m}^{-2}\right)$, precipitation rate $\left(P R, \mathrm{~mm} \mathrm{month}^{-1}\right)$, relative humidity $(\mathrm{RH}, \%)$, temperature at $925 \mathrm{hPa}$ (Temp, K) and surface wind speed (WS, $\left.\mathrm{m} \mathrm{s}^{-1}\right)$; and aerosol burden measured as aerosol optical depth (AOD, unit less) over Lahore during October 2004 September 2015

\begin{tabular}{c|c|c|c|c|c|c|c|c|c|c|c|c}
\hline Parameter & Tropo $\mathbf{C H}_{4}$ & Tropo CO & Tropo $\mathbf{C O}_{2}$ & ${\text { Tropo } \mathbf{N O}_{2}}$ & Tropo $\mathbf{O}_{3}$ & Tropo $\mathbf{S O}_{2}$ & AOD & CLF & OLR & PR & RH & Temp \\
\hline TropoCO & 0.11 & & & & & & & & & & & \\
\hline TropoCO $_{2}$ & $\mathbf{0 . 0 0}$ & $\mathbf{0 . 0 1}$ & & & & & & & & & & \\
\hline TropoNO $_{2}$ & 0.08 & 0.94 & $\mathbf{0 . 0 0}$ & & & & & & & & & \\
\hline TropoO $_{3}$ & 0.39 & $\mathbf{0 . 0 0}$ & 0.12 & 0.50 & & & & & & & & \\
\hline TropoSO $_{2}$ & 0.77 & 0.13 & 0.36 & 0.03 & $\mathbf{0 . 0 1}$ & & & & & & & \\
\hline AOD & $\mathbf{0 . 0 0}$ & 0.02 & 0.95 & 0.41 & 0.31 & 0.89 & & & & & & \\
\hline CLF & $\mathbf{0 . 0 0}$ & 0.06 & 0.91 & 0.30 & 0.44 & 0.01 & 0.03 & & & & & \\
\hline OLR & 0.41 & $\mathbf{0 . 0 0}$ & 0.51 & 0.09 & $\mathbf{0 . 0 0}$ & 0.58 & 0.01 & $\mathbf{0 . 0 0}$ & & & & \\
\hline PR & $\mathbf{0 . 0 0}$ & 0.06 & 0.83 & 0.09 & 0.97 & 0.17 & $\mathbf{0 . 0 0}$ & $\mathbf{0 . 0 0}$ & 0.26 & & & \\
\hline RH & $\mathbf{0 . 0 0}$ & 0.18 & 0.76 & 0.08 & $\mathbf{0 . 0 0}$ & 0.68 & $\mathbf{0 . 0 0}$ & $\mathbf{0 . 0 0}$ & $\mathbf{0 . 0 0}$ & $\mathbf{0 . 0 0}$ & & \\
\hline Temp & $\mathbf{0 . 0 0}$ & 0.07 & 0.22 & 0.11 & $\mathbf{0 . 0 0}$ & 0.97 & $\mathbf{0 . 0 0}$ & 0.29 & $\mathbf{0 . 0 0}$ & $\mathbf{0 . 0 0}$ & 0.22 & \\
\hline WS & 0.15 & $\mathbf{0 . 0 0}$ & $\mathbf{0 . 0 0}$ & $\mathbf{0 . 0 1}$ & $\mathbf{0 . 0 0}$ & 0.13 & 0.63 & $\mathbf{0 . 0 0}$ & 0.27 & 0.12 & 0.03 & 0.22 \\
\hline
\end{tabular}

The numbers written in bold text are statistical significance values for significant correlations at the 0.01 level (2-tailed). The numbers written in italic text are statistical significance values for significant correlations at the 0.05 level (2-tailed)

Annual trends (slopes and y-intercepts), overall changes (\%), and $\mathrm{R}^{2}$ of tropospheric gases, meteorological parameters, and aerosol optical depth over Lahore using their annual-means from October 2004 to September 2015 are tabulated in Table 4. All tropospheric gases except TropoCO indicate an increasing trend. Maximum positive 
slope of $4.93 \pm 0.18$ with $\mathrm{R}^{2}=0.99$ observed for $\mathrm{TropoCH}_{4}$ (having high correlations [0.54] significant at 0.01 level with $\mathrm{TropoCO}_{2}$ and $\mathrm{RH}$ ) indicates a maximum increasing trend than other gases used for this study. Similarly, slopes of PR (3.21 \pm 1.08$)$ with maximum change of $49.61 \%$ (having strong correlation with CLF), and $\mathrm{TropoCO}_{2}(1.97 \pm 0.04$, having high correlation with TropoCH${ }_{4}$ ) show relatively increasing trends during the study period. However, a relatively large negative slope of TropoCO $(-3.48 \pm 0.54)$ shows a decreasing trend over Lahore, which may be due to an overall decrease in TropoCO $(-23.8 \%)$ during the entire study period. Moreover, TropoCO has inverse but high correlations significant at 0.01 level with $\mathrm{TropoO}_{3}$ and WS. Relatively higher positive slopes $(\sim 21 \%)$ have been noticed for TropoNO $\mathrm{N}_{2}, \mathrm{CLF}$, and WS, whereas little negative slopes have been observed for OLR $(-0.05 \pm 0.21)$ and temperature $(-0.10 \pm 0.04)$. It is worth mentioning here that the monthly-mean temperature values over Lahore during October 2004 - September 2015 show a little increasing trend (Figure 3 refers).

Table 4. Annual trends (linear equation showing slopes and y-intercepts), overall change (\%), and $R^{2}$ based on annual-means of tropospheric gases including TropoCH, TropoCO, TropoCO $\mathrm{C}_{2}$, TropoNO $\mathrm{N}_{2}$ and TropoSO $\mathrm{S}_{2}$; major meteorological parameters including cloud fraction $(C L F)$, outgoing long-wave radiation $(O L R)$, precipitation rate $(P R)$, relative humidity (RH), temperature (Temp) and surface wind speed (WS); and aerosol burden measured as aerosol optical depth (AOD) over Lahore during October 2004-September 2015

\begin{tabular}{|c|c|c|c|}
\hline Parameter & Annual Trend Line & Overall Change (\%) & $R^{2}$ \\
\hline $\mathrm{TropoCH}_{4}$ (ppbv) & $y=(4.93 \pm 0.18) x+(1799.10 \pm 1.35)$ & 2.92 & 0.99 \\
\hline TropoCO (ppbv) & $y=-(3.48 \pm 0.54) x+(202.36 \pm 3.94)$ & -23.80 & 0.81 \\
\hline $\mathrm{TropoCO}_{2}(\mathrm{ppm})$ & $y=(1.97 \pm 0.04) x+(377.07 \pm 0.27)$ & 5.40 & 1.00 \\
\hline TropoNO $_{2}\left(\times 10^{15}\right.$ molecules $\left.\mathrm{cm}^{-2}\right)$ & $y=(0.06 \pm 0.02) x+(2.23 \pm 0.17)$ & 21.62 & 0.40 \\
\hline $\mathrm{TropoO}_{3}(\mathrm{ppbv})$ & $y=(0.29 \pm 0.08) x+(47.91 \pm 0.62)$ & 6.18 & 0.54 \\
\hline $\mathrm{TropoSO}_{2}(\mathrm{DU})$ & $y=(0.00 \pm 0.00) x+(0.06 \pm 0.02)$ & 13.27 & 0.01 \\
\hline CLF (unit less) & $y=(0.00 \pm 0.00) x+(0.19 \pm 0.02)$ & 21.47 & 0.29 \\
\hline OLR $\left(\mathrm{W} \mathrm{m}^{-2}\right)$ & $y=-(0.05 \pm 0.21) x+(302.66 \pm 1.52)$ & -0.18 & 0.01 \\
\hline PR (mm month $\left.{ }^{-1}\right)$ & $y=(3.21 \pm 1.08) x+(32.62 \pm 7.97)$ & 49.61 & 0.47 \\
\hline $\mathrm{RH}(\%)$ & $y=(0.41 \pm 0.15) x+(40.71 \pm 1.13)$ & 9.94 & 0.42 \\
\hline Temp (K at $925 \mathrm{hPa}$ ) & $y=-(0.10 \pm 0.04) x+(300.50 \pm 0.30)$ & -0.37 & 0.38 \\
\hline WS $\left(\mathrm{m} \mathrm{s}^{-1}\right)$ & $y=(0.05 \pm 0.01) x+(1.86 \pm 0.08)$ & 21.43 & 0.66 \\
\hline AOD (unit less) & $y=(0.01 \pm 0.00) x+0.62 \pm 0.02$ & 10.69 & 0.39 \\
\hline
\end{tabular}

\section{Results and Discussion}

\section{Parametric out-look of Lahore}

Tropospheric gases

Concentrations of major tropospheric gases have been compared with those of $\mathrm{TropoO}_{3}$ over Lahore during October 2004 - September 2015 and are shown in Figure 2. Large variations have been observed in $\mathrm{TropoCH}_{4}$, TropoCO, and $\mathrm{TropoNO}_{2}$, whereas, Tropo $\mathrm{CO}_{2}$ and $\mathrm{TropoSO}_{2}$ show comparatively little variations. We also noticed that all tropospheric gases indicate increasing trends except TropoCO. Gradual increasing trend in $\mathrm{TropoCH}_{4}$ indicates gradual increasing activities responsible for Methane production (Paddy crops, animals, industrial activities, and vehicular emissions etc.) in the study area 
during the study period. Similarly, gradual increasing trend in $\mathrm{TropoCO}_{2}$ is an indication of increased activities causing carbon dioxide to increase (Agricultural activities, population, animals, industrial activities, and vehicular emissions etc.) in Lahore. Whereas, slopes of $\mathrm{TropoNO}_{2}$ and $\mathrm{TropoSO}_{2}$ are quite gentle showing little increase similar to that of $\mathrm{TropoO}_{3}$.

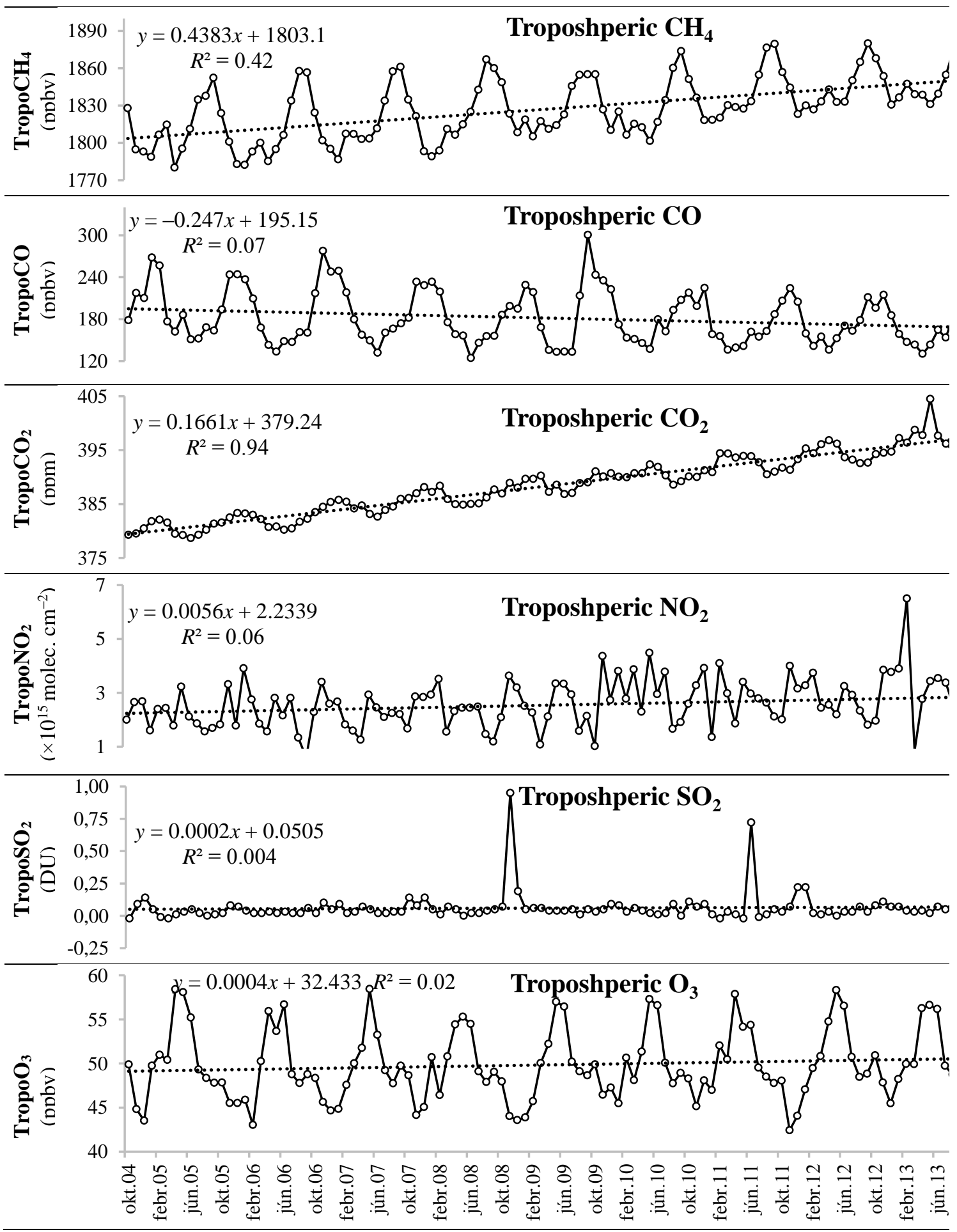

Figure 2. Time series monthly-mean variations of major tropospheric gases over Lahore during October 2004 - September 2015. Dotted lines correspond to respective trend lines 


\section{Meteorological parameters}

Monthly-mean data products of major meteorological parameters as well as aerosol burden measured as Aerosol Optical Depth (AOD) have been compared with TropoO 3 concentrations over Lahore during October 2004 - September 2015 and are shown in Figure 3. Large variations have been observed in all parameters similar to that of $\mathrm{TropoO}_{3}$ during the entire study period. Positive slopes of all parameters including $\mathrm{TropoO}_{3}$, except OLR, indicate increasing trends during the study period. Comparatively larger slope value of PR indicates a gradual increase in precipitation in the study area.

\section{$\mathrm{TropoO}_{3}$}

Maximum concentration of $\mathrm{TropoO}_{3}$ has been reported during spring and summer seasons across the globe (e.g., Cooper et al., 2014). Accordingly, maximum concentrations have been observed in $\mathrm{TropoO}_{3}$ over Lahore during spring and summer (monsoon) seasons (March to July) in Pakistan shown in Figure 4. Major causes of this increased $\mathrm{TropoO}_{3}$ concentration over Lahore are mainly due to increased ozone precursor gas emissions, enhanced biomass burning activities, biogenic emissions, polluted air-masses migrated from nearby regions, removal of $\mathrm{NO}_{2}$ concentration due to increased $\mathrm{OH}$ radicalization (Yienger and Levy, 1995), photolysis of water vapors which reduces $\mathrm{NO}_{2}$ concentration during high rainfall in monsoon (Jacob, 2003; Ghude et al., 2009; Seinfeld and Pandis, 2016), and enhanced intrusion of $\mathrm{O}_{3}$ from stratosphere (Noreen et al., 2018). An overall decreasing trend has been observed in the monthly-mean values of observed $\mathrm{TropoO}_{3}$ concentration over Lahore (slope $=-0.4303$, $\mathrm{y}$-intercept $=52.786$, and $\mathrm{R}^{2}=0.1614$ ) during the study period.

\section{Modeling $\mathrm{TropoO}_{3}$ concentration over Lahore}

In order to investigate $\mathrm{TropoO}_{3}$ dependencies, monthly-mean values of major tropospheric gases, meteorological parameters, and aerosol burden over Lahore during October 2004 - September 2015 have been incorporated in multiple linear regression model (e.g., Engel-Cox et al., 2004; Lin et al., 2012), mentioned in Equation (1);

$$
\text { Objective or Dependent Variable }=\mathrm{a}_{\mathrm{o}}+\sum\left(\mathrm{b}_{\mathrm{n}} \times \mathrm{w}_{\mathrm{n}}\right)+\varepsilon
$$

where $a_{0}$ is an intercept, $b=1,2,3, \ldots . n$ are regular (unstandardized) regression coefficients, $\mathrm{w}=1,2,3, \ldots \ldots \mathrm{n}$ are regressors or independent variables, and $\varepsilon$ is an error term associated with the regression analysis. The resultant multiple linear regression model used in this study to model $\mathrm{TropoO}_{3}$ (Objective/Dependent Variable) as functions of tropospheric gases, meteorological parameters, and aerosol burden (regressors or independent variables) is mentioned in Equation (2) below;

$$
\begin{gathered}
\text { TropoO }_{3} \text { (monthly mean) } \\
=\mathrm{a}_{0}+\mathrm{b}_{1} \times \text { TropoCH }_{4} \\
+\mathrm{b}_{2} \times \text { TropoCO } \\
+\mathrm{b}_{3} \times \text { TropoCO }_{2}+ \\
\mathrm{b}_{4} \times \text { TropoNO }_{2}+\mathrm{b}_{5} \times \text { TropoSO }_{2}+\mathrm{b}_{6} \times \mathrm{CLF} \\
+\mathrm{b}_{7} \times \mathrm{OLR}+ \\
\mathrm{b}_{8} \times \mathrm{PR}+\mathrm{b}_{9} \times \mathrm{RH}+\mathrm{b}_{10} \times \mathrm{Temp}+\mathrm{b}_{11} \times \mathrm{WS} \\
+\mathrm{b}_{12} \times \text { AOD }+\varepsilon
\end{gathered}
$$


where $a_{0}$ is an intercept, $b_{1}, b_{2}, b_{3}, \ldots \ldots b_{12}$ are regular (unstandardized) regression coefficients; TropoCH $\mathrm{CH}_{4}$, TropoCO, TropoCO $\mathrm{C}_{2}, \mathrm{TropoNO}_{2}, \mathrm{TropoSO}_{2}, \mathrm{CLF}, \mathrm{OLR}, \mathrm{PR}$, $\mathrm{RH}$, Temp, WS, and AOD are the monthly-mean regressors or independent variables; and $\varepsilon$ is an error term associated with the regression analysis. The multiple linear regression model mentioned in Equation (2) closely measures the intrinsic relationships of TropoO 3 with all regressors, separately. Therefore, this model offers better predictability of $\mathrm{TropoO}_{3}$ based on relative impacts with all regressors than by simple linear model which is based only upon single regressor.

To improve the results of Equation (2), stepwise multiple linear regression procedure (Lin et al., 2012) was adopted. Resultant stepwise multiple linear regression model shown in Table 5 was generated. The model provides statistical information for modeling $\mathrm{TropoO}_{3}$ concentrations based upon weighted contributions from its regressors. This procedure simultaneously regresses multiple variables (gases, meteorological parameters, and aerosol burden) and removes un-important parameter(s) from the model. Table 5 shows that the modeled $\mathrm{TropoO}_{3}$ is mainly dependent upon OLR, WS, CLF, TropoSO TropoCO, and RH out of all regressors mentioned in Equation (2). The standardized regression coefficients $(\beta)$ obtained from the stepwise multiple linear regression model show weight-wise dependence of $\mathrm{TropoO}_{3}$ upon OLR $[\beta=0.458]$ with the highest contribution, followed by nearly equivalent contributions from WS [ $\beta=0.262]$ and CLF [ $\beta=0.256]$, whereas, the least contribution has been observed from $\mathrm{RH}[\beta=-0.320]$. This model also reveals that $\mathrm{TropoSO}_{2}[\beta=-0.097]$ and TropoCO $[\beta=-0.231]$ are negatively contributing against $\mathrm{TropoO}_{3}$ concentration over Lahore during the study period. The values of multiple error $(r=0.85)$ and standard error (2.14) for this $\mathrm{TropoO}_{3}$ model appear to be reasonable.

A subsequent bivariate correlation matrix representing observed $\mathrm{TropoO}_{3}$, modeled $\mathrm{TropoO}_{3}$, and the parameters which contributed in the modeled $\mathrm{TropoO}_{3}$ concentration (OLR, WS, CLF, TropoSO $\mathrm{S}_{2}$, TropoCO, and $\mathrm{RH}$ ) as mentioned in Table 5 above was generated and is shown in Table 6 below. Blue color indicates positive correlations, while red color indicates negative/inverse correlations. Longer the length of colored bars (either blue or red), stronger would be the correlation (either positive or inverse, respectively) between the corresponding parameters. In Table 6, correlation values written in bold text are significant at the 0.01 level (2-tailed), whereas, in italic text are significant at the 0.05 level (2-tailed). Respective statistical significance values, against each correlations of Table 6, are written in Table 7. The highest statistical significance values at the 0.01 level (2-tailed) are highlighted in bold text, whereas, the statistical significance values at the 0.05 level (2-tailed) are mentioned in italic text in Table 7. The values written in regular text indicate least significance values. All correlations shown in Table 6 exhibit the same corresponding values as mentioned in Table 2 above along with additive correlation values against modeled $\mathrm{TropoO}_{3}$. All bivariate correlations with the modeled $\mathrm{TropoO}_{3}$ concentration are significant at 0.01 level (2-tailed) except for CLF. The highest correlation value [0.85] with $99 \%$ significance at 0.00 level (2-tailed) between modeled and observed $\mathrm{TropoO}_{3}$ concentrations over Lahore during the study period reveals the high suitability of the modeled developed in this study mentioned in Table 5 above. Out of two tropospheric gases used in this model, modeled $\mathrm{TropoO}_{3}$ is found to be highly but inversely correlated with TropoCO [-0.72], whereas, OLR [0.71] and WS [0.68] are the next highly and positively correlated meteorological parameters significant at 0.01 level (2-tailed). 


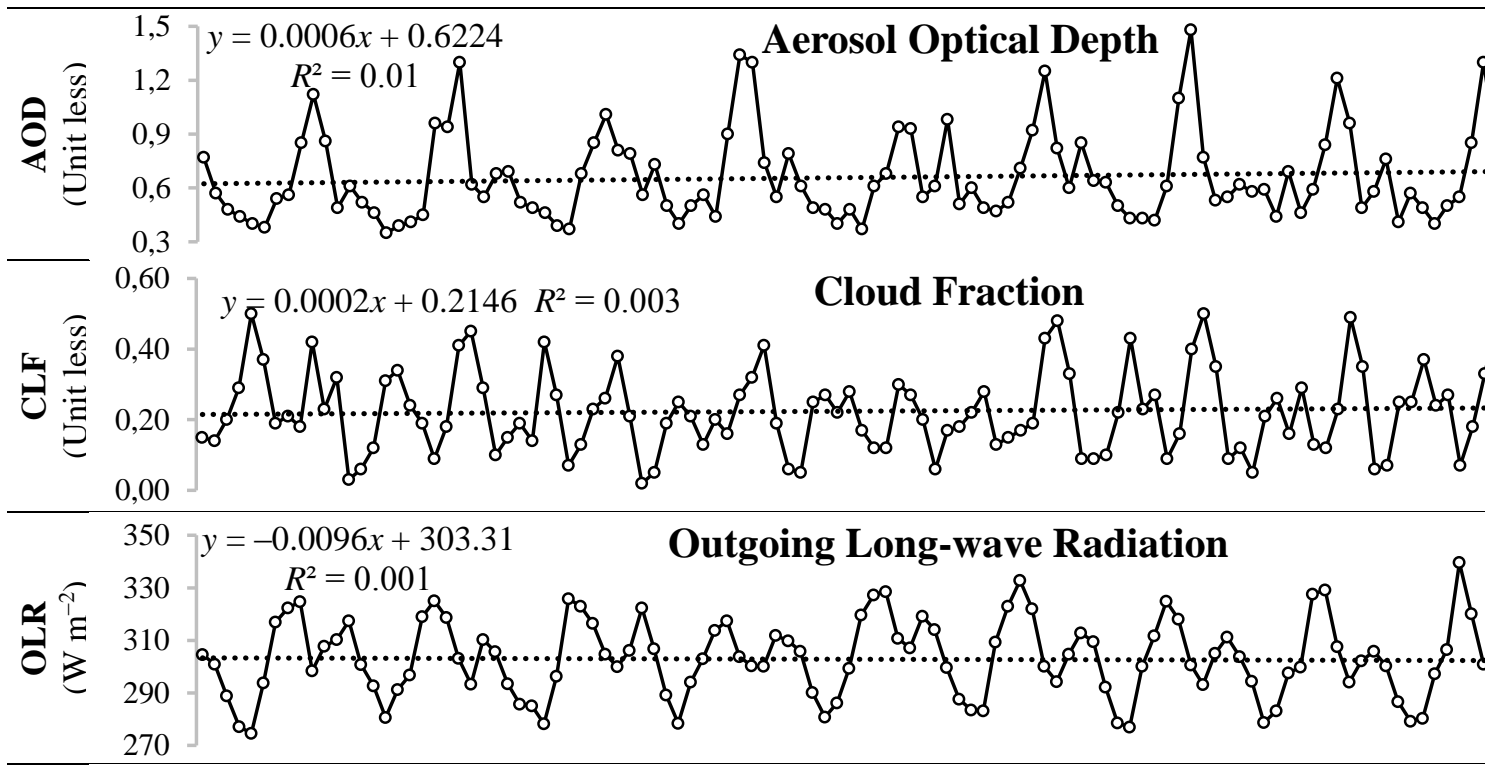

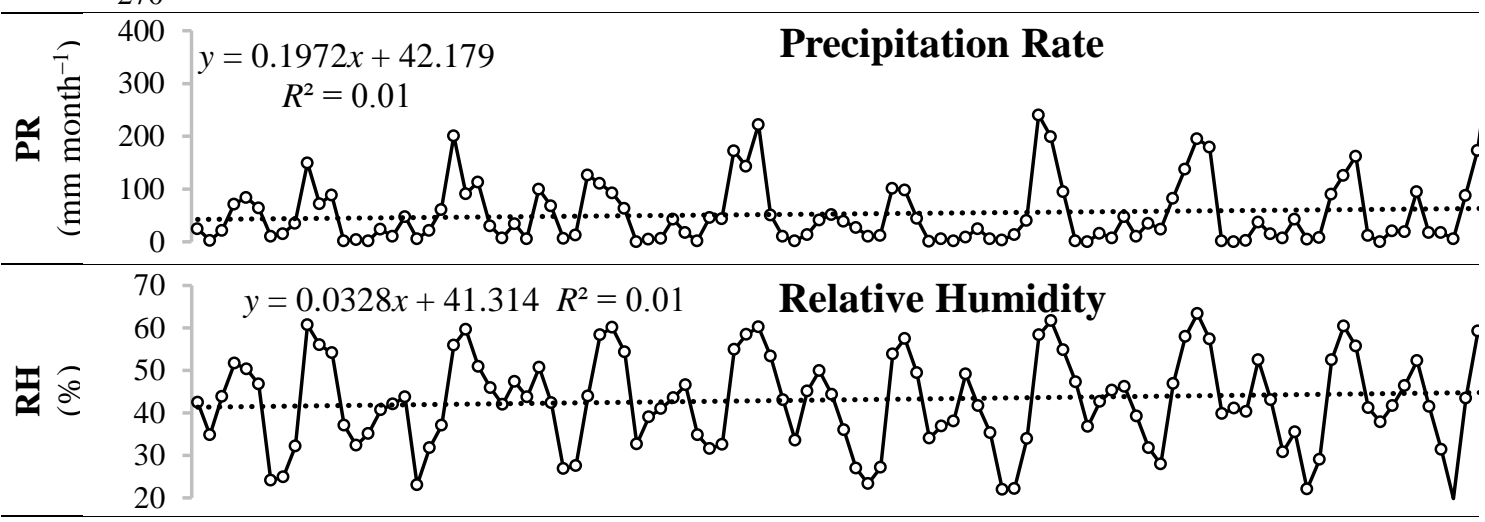
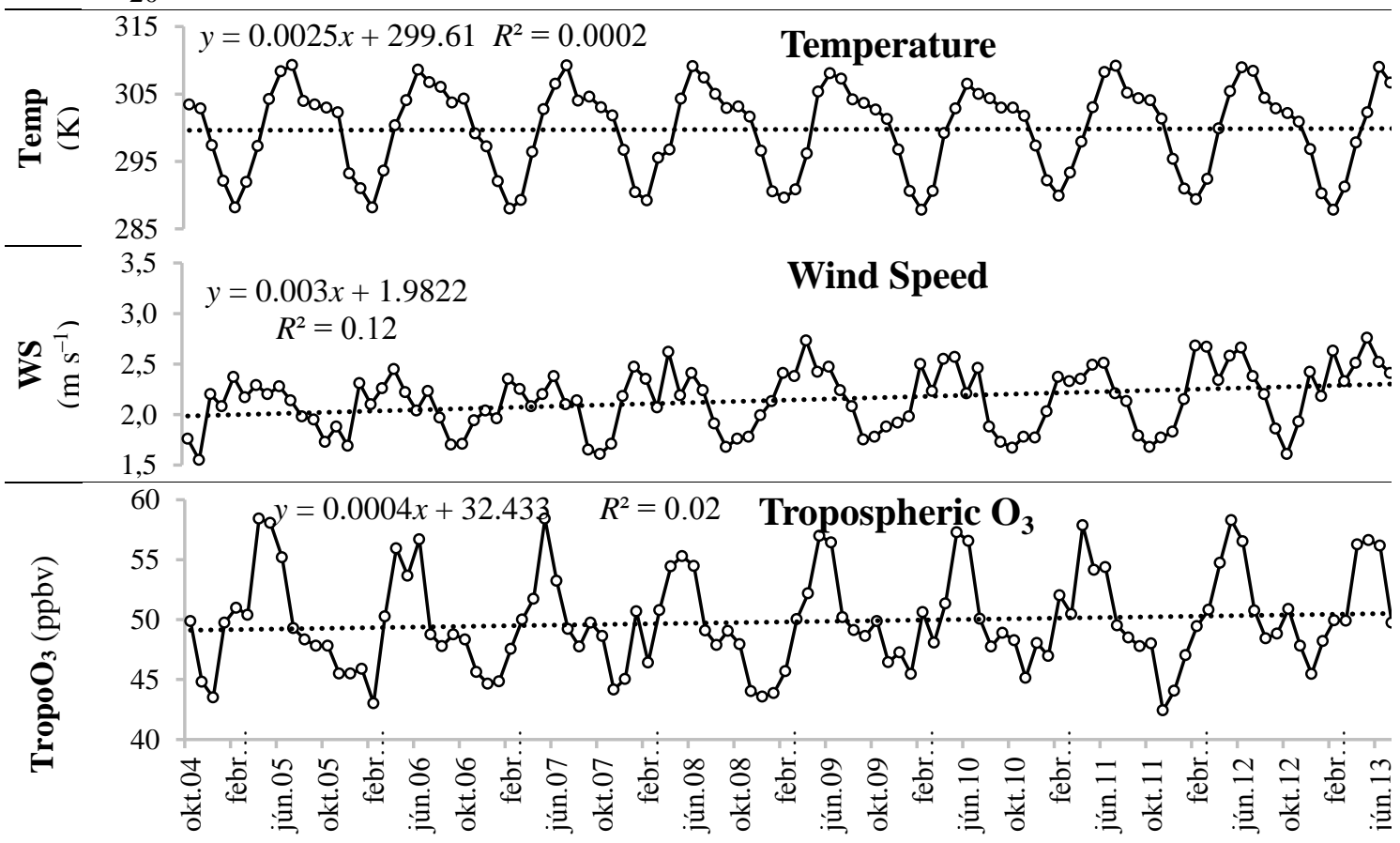

Figure 3. Time series monthly-mean variations of Tropo $\mathrm{O}_{3}$ and major meteorological parameters over Lahore during October 2004 - September 2015. Dotted lines correspond to respective trend lines 


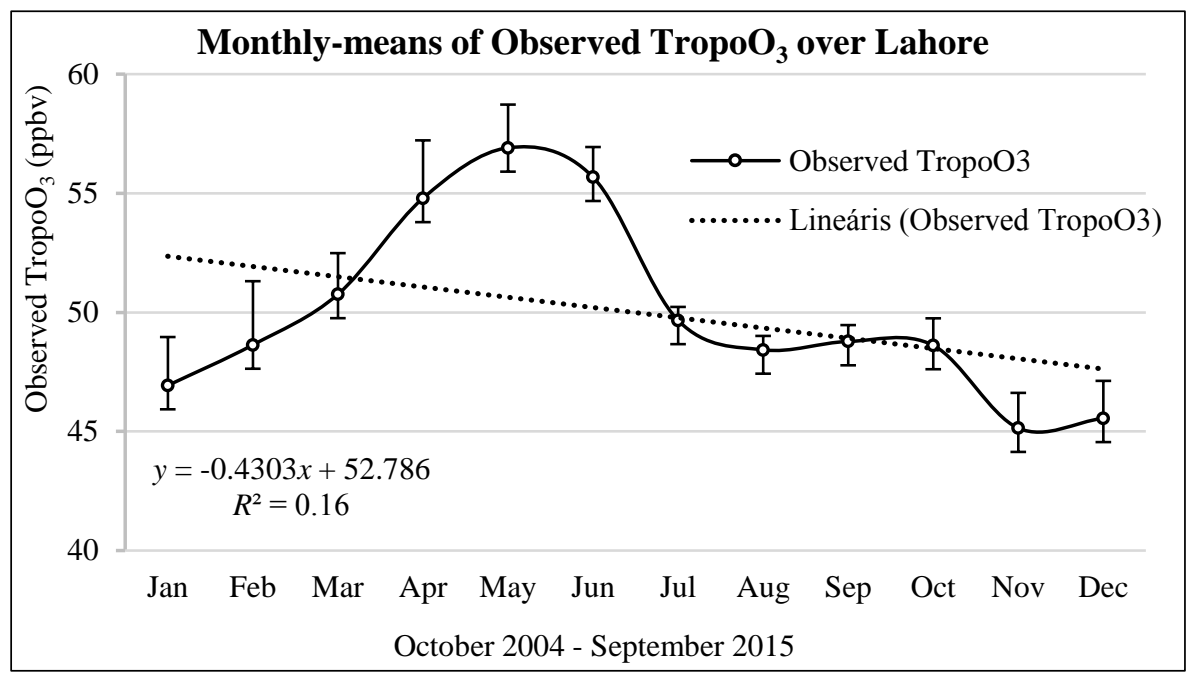

Figure 4. Monthly-means of Observed TropoO 3 concentration (ppbv) over Lahore during October 2004 - September 2015. Dotted line corresponds to the trend line

Table 5. Stepwise multiple linear regression model developed to model TropoO 3 (ppbv) variations based upon weighted contributions of monthly-mean data of OLR $\left(W^{-2}\right), W S(m$ $s^{-1}$ ), CLF (unit less), TropoSO $\mathrm{O}_{2}(\mathrm{DU})$, TropoCO (ppbv), and $\mathrm{RH}(\%)$ over Lahore during October 2004 - September 2015

\begin{tabular}{l|c|c}
\multicolumn{1}{c|}{ Stepwise multiple linear regression model equation } & Multiple $\boldsymbol{r}$ & $\begin{array}{c}\text { Standard } \\
\text { Error }\end{array}$ \\
\hline $\begin{array}{l}\mathrm{TropoO}_{3}(\text { monthly-mean })=12.706+0.127 O L R[0.458]+3.213 W S[0.262]+ \\
9.078 C L F[0.256]-3.648 \text { TropoSO }_{2}[-0.097]-0.026 \text { TropoCO}[-0.231]-0.121 R H[-\end{array}$ & 0.85 & 2.14 \\
$0.320]$ & & \\
\hline
\end{tabular}

The values in parentheses are Standardized Regression Coefficients (Beta, $\beta$ ) representing regressor's relative weightage on modeled $\mathrm{TropoO}_{3}$ concentrations in descending order, although these coefficients do not take part in calculations

Table 6. Bivariate correlation matrix representing monthly-mean data of observed $\mathrm{TropoO}_{3}$, $\mathrm{OLR}\left(\mathrm{W} \mathrm{m}^{-2}\right), \mathrm{WS}\left(\mathrm{m} \mathrm{s}^{-1}\right), \mathrm{CLF}$ (unit less), TropoSO 2 (DU), TropoCO (ppbv), and $\mathrm{RH}(\%)$ as well as modeled TropoO $\mathrm{O}_{3}$ concentrations over Lahore during October 2004 - September 2015

\begin{tabular}{|c|c|c|c|c|c|c|c|}
\hline Parameters & $\begin{array}{c}\text { Observed } \\
\text { TropoO }_{3}\end{array}$ & OLR & WS & CLF & TropoSO $_{2}$ & TropoCO & RH \\
\hline OLR & \begin{tabular}{|l|l|}
0.61 \\
\end{tabular} & & & & & & \\
\hline WS & 0.57 & 0.10 & & & & & \\
\hline CLF & -0.07 & -0.48 & 0.25 & & & & \\
\hline TropoSO$_{2}$ & -0.23 & -0.05 & -0.13 & 0.22 & & & \\
\hline TropoCO & -0.61 & -0.31 & -0.54 & -0.17 & 0.13 & & \\
\hline RH & -0.46 & -0.52 & 0.19 & 0.70 & -0.03 & 0.12 & \\
\hline Modeled TropoO $_{3}$ & 0.85 & 0.71 & 0.68 & -0.07 & -0.27 & -0.72 & -0.53 \\
\hline
\end{tabular}

Correlations in bold text are significant at the 0.01 level (2-tailed). Correlations in Italic are significant at the 0.05 level (2-tailed). Blue color indicates positive correlations, and red color indicates negative/inverse correlations. Longer the length of colored bars (either blue or red), stronger would be the correlation (either positive or inverse, respectively) between the corresponding parameters 


$$
-13545 \text { - }
$$

Table 7. Based upon correlation matrix discussed in Table 6 above, matrix showing statistical significance values of observed TropoO ${ }_{3}, \mathrm{OLR}\left(\mathrm{W} \mathrm{m}^{-2}\right), \mathrm{WS}\left(\mathrm{m} \mathrm{s}^{-1}\right), \mathrm{CLF}$ (unit less), TropoSO $\mathrm{S}_{2}$ $(\mathrm{DU})$, TropoCO (ppbv), and $\mathrm{RH}(\%)$ as well as modeled Tropo $\mathrm{O}_{3}$ concentrations over Lahore during October 2004 - September 2015

\begin{tabular}{|c|c|c|c|c|c|c|c|}
\hline Parameters & $\begin{array}{c}\text { Observed } \\
\text { TropoO }\end{array}$ & OLR & WS & CLF & $\mathrm{TropoSO}_{2}$ & TropoCO & RH \\
\hline OLR & 0.00 & & & & & & \\
\hline WS & 0.00 & 0.27 & & & & & \\
\hline CLF & 0.44 & 0.00 & 0.00 & & & & \\
\hline TropoSO $_{2}$ & 0.01 & 0.58 & 0.13 & 0.01 & & & \\
\hline TropoCO & 0.00 & 0.00 & 0.00 & 0.06 & 0.13 & & \\
\hline RH & 0.00 & 0.00 & 0.03 & 0.00 & 0.68 & 0.18 & \\
\hline Modeled TropoO & 0.00 & 0.00 & 0.00 & 0.42 & 0.00 & 0.00 & 0.00 \\
\hline
\end{tabular}

The numbers written in bold text are statistical significance values for significant correlations at the 0.01 level (2-tailed). The numbers written in italic text are statistical significance values for significant correlations at the 0.05 level (2-tailed)

Other inverse correlations of the modeled $\mathrm{TropoO}_{3}$ significant at 0.00 level (2-tailed) are noticed against $\mathrm{RH}[-0.53]$ and $\mathrm{TropoSO}_{2}[-0.27]$. The least and inverse correlation having 95\% significance at 0.05 level (2-tailed) is against CLF [-0.07].

The observed $\mathrm{TropoO}_{3}$ datasets retrieved directly from the AIRS/AMSU-A sensor over Lahore during October 2004 - September 2015 and the modeled TropoO 3 concentration values obtained from of the resultant step-wise multiple linear regression model (discussed in Table 5 above) have been plotted in Figure 5.

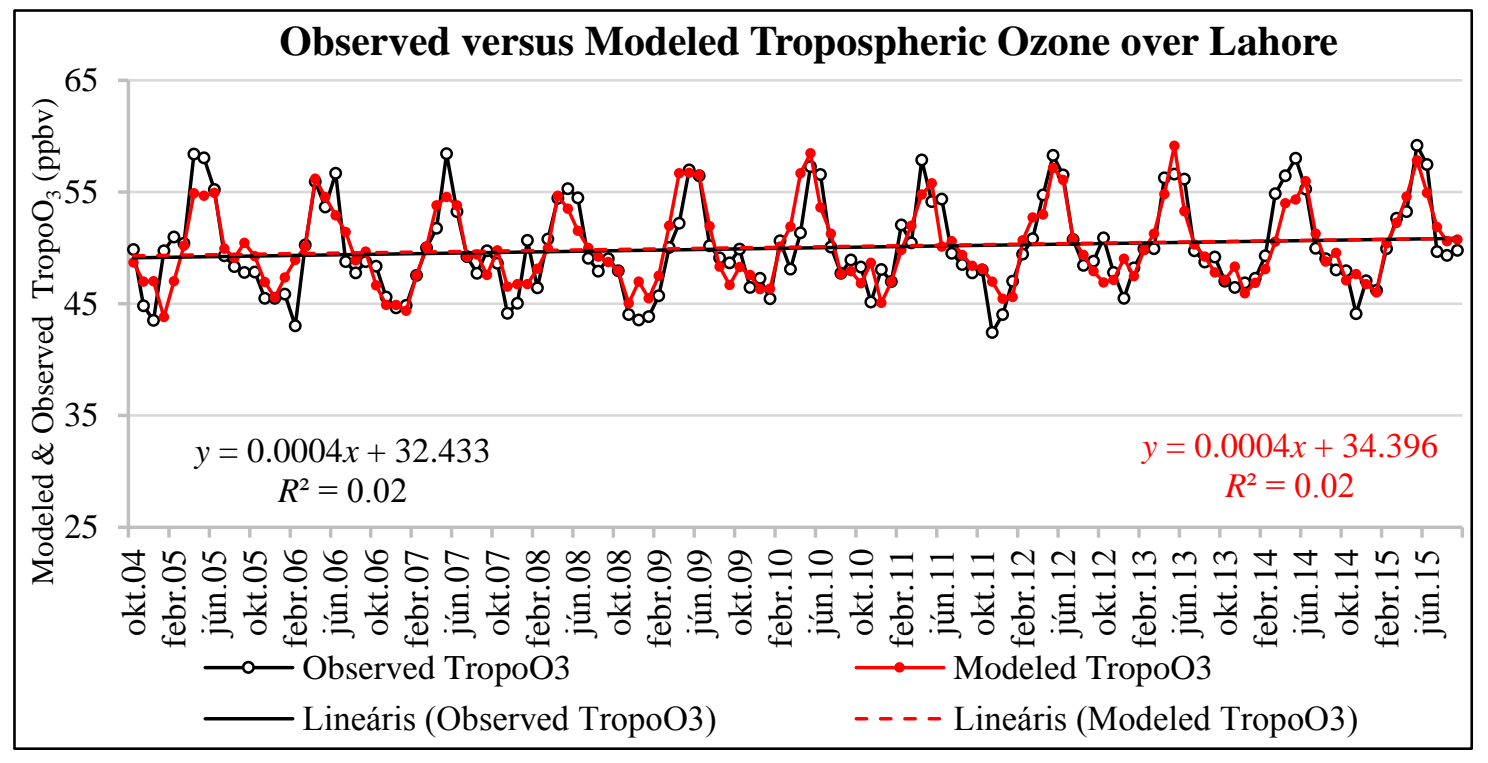

Figure 5. Comparison of observed versus modeled time-series $\mathrm{TropoO}_{3}$ (ppbv) concentrations over Lahore during October 2004 - September 2015. Dotted lines correspond to respective trend lines

Time series distributions of these observed and modeled $\mathrm{TropoO}_{3}$ concentrations show similar increasing and consistent trends except during the months of May 2005, Feb 2006, May 2007, Dec 2008, Nov 2011, Dec 2012, May 2014, and Nov 2014. The highest value in the modeled $\mathrm{TropoO}_{3}$ concentration has been notice for the month of May 2013. 
Monthly-mean data analysis of modeled $\mathrm{TropoO}_{3}$ concentration indicate a similar pattern as that of the observed $\mathrm{TropoO}_{3}$ concentration over Lahore during the study period as shown in Figure 6(a). We find higher concentrations of both modeled and observed $\mathrm{TropoO}_{3}$ during spring and summer (monsoon) seasons, whereas, minima during the winter season. A similar observation about $\mathrm{TropoO}_{3}$ variation was reported by Ahmad and Aziz, 2013. Our modeled $\mathrm{TropoO}_{3}$ variation attributes a similar decreasing trend (slope $=-0.3254$, $\mathrm{y}$-intercept $=52.192$, and $\mathrm{R}^{2}=0.12$ ) over Lahore during the study period. Scatter plot drawn between observed and modeled $\mathrm{TropoO}_{3}$ indicates a linear positive trend having a slope 0.732 and y-intercept 13.469 with $\mathrm{R}^{2}=0.73$ as shown in Figure $6(b)$.
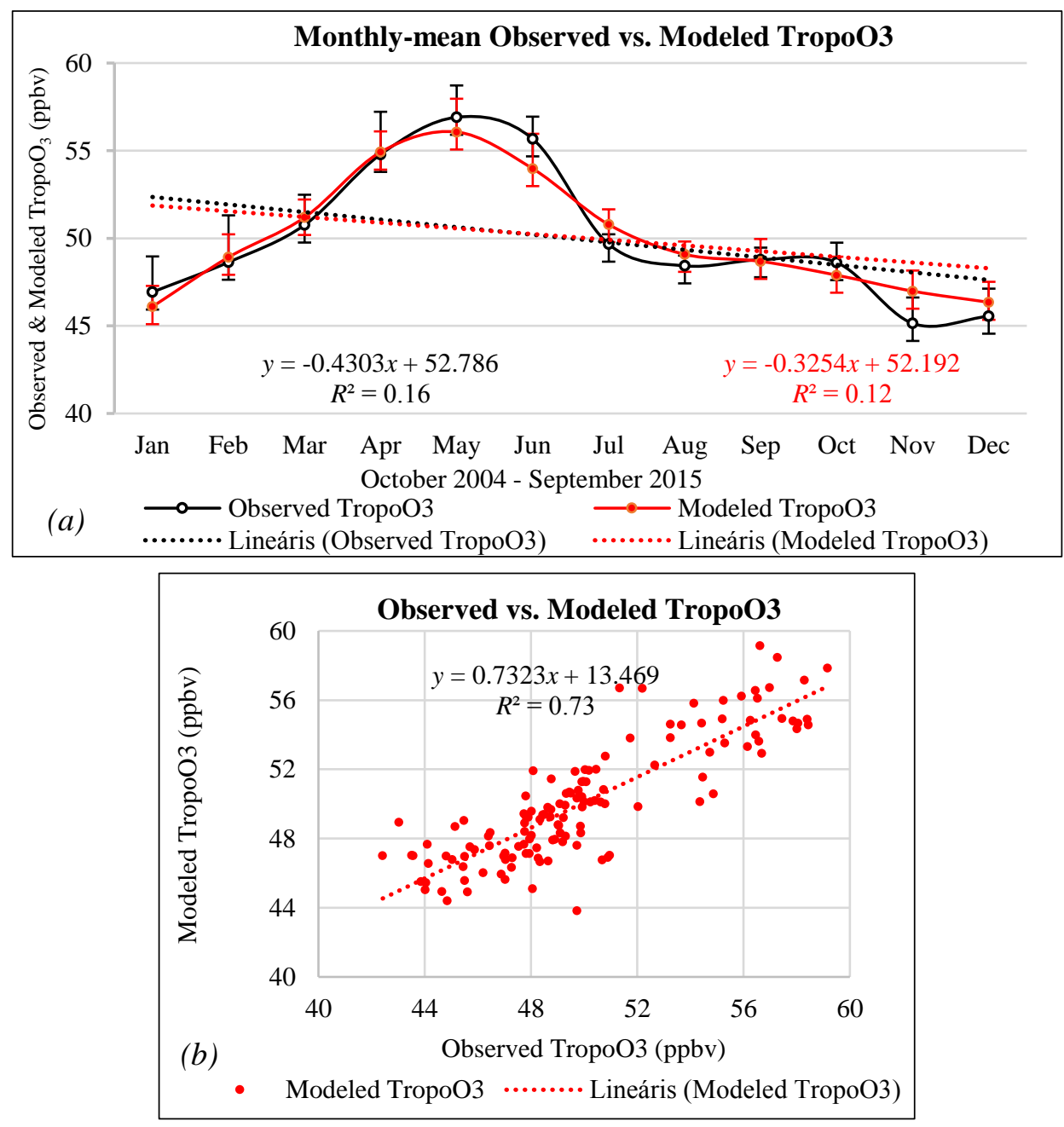

Figure 6. (a) Monthly-means of observed TropoO $\mathrm{O}_{3}$ (used as dependent variable) and modeled TropoO $O_{3}$ concentrations (all data in ppbv) over Lahore during October 2004 - September 2015. (b) Scatter plot of observed versus modeled TropoO $O_{3}$ concentrations (ppbv). Dotted lines correspond to respective trend lines

Annual trends, overall change $(\%)$, and $\mathrm{R}^{2}$ based on annual-means of observed and modeled $\mathrm{TropoO}_{3}$ concentrations over Lahore during October 2004 - September 2015 were calculated and are mentioned in Table 8. 
Table 8. Annual trends (slopes and y-intercepts), overall change (\%), and $R^{2}$ based on annualmeans of observed and modeled TropoO $\mathrm{O}_{3}$ concentrations over Lahore during October 2004 September 2015

\begin{tabular}{c|c|c|c}
\hline Parameter & Annual Trend Line & Overall Change (\%) & $\boldsymbol{R}^{\mathbf{2}}$ \\
\hline${\text { Observed } \mathrm{TropoO}_{3}(\mathrm{ppbv})}^{\text {Modeled } \mathrm{TropoO}_{3}(\mathrm{ppbv})}$ & $y=(0.29 \pm 0.08) x+(47.91 \pm 0.62)$ & 6.18 & 0.54 \\
& $y=(0.23 \pm 0.06) x+(48.48 \pm 0.42)$ & 4.89 & 0.62 \\
\hline
\end{tabular}

Overall similar increasing concentrations of both observed $\left(6.18 \%, R^{2}=0.54\right)$, and modeled $\left(4.89 \%, R^{2}=0.62\right) \mathrm{TropoO}_{3}$ indicate good agreement of resultant model devised in this study. The slope of observed $\mathrm{TropoO}_{3}[0.29 \pm 0.08]$ is little larger than that of the observed $\mathrm{TropoO}_{3}[0.23 \pm 0.06]$, however, the values of y-intercepts of both concentrations are nearly similar. The increase in $\mathrm{TropoO}_{3}$ concentration observed in Lahore and adjoining areas during the study period is possibly due to increased anthropogenic activities including; population and traffic densities, industrial activities, infrastructure development activities, and biomass burning etc. These results are almost in agreement with those obtained by Noreen et al 2018 for ozone column over Lahore. Some other studies had also discussed about the $\mathrm{TropoO}_{3}$ concentrations, and production of its precursors through different anthropogenic activities (e.g., Kulkarni et al., 2010; Lal et al., 2012; Ahmad and Aziz, 2013).

\section{Model validation}

Modeled $\mathrm{TropoO}_{3}$ concentrations were crossed checked against an independent source of TropoO $\mathrm{O}_{3}$ concentrations. For this purpose, original $\mathrm{TropoO}_{3}$ data product of subtracted monthly-means of Total Column Ozone retrievable from OMI and Stratospheric Column Ozone retrievable from MSL onboard Aura satellite was utilized (available at https://acdext.gsfc.nasa.gov/Data_services/cloud_slice/new_data.html). $\quad \mathrm{TropoO}_{3} \quad$ product calculated by mean volume mixing ratio (in ppbv) formatted in 1-degree (latitude) by 1.25-degree (longitude) resolution was extracted for Lahore during October 2004 September 2015. Our modeled as well as the observed TropoO $_{3}$ (used as dependent variable) concentrations have been plotted in Figure 7 against these independently available product of OMI-MLS TropoO 3 concentrations (ppbv) over Lahore during the study period.

The results show similar seasonality patterns as well as trends in all three datasets with good agreements in their slopes and error values mentioned in Figure 7. These promising results validate our resultant model. OMI-MLS TropoO ${ }_{3}$ concentrations have been plotted against secondary axis due to its wider data range. OMI/MLS data measurements have some anomalies especially during 2007 and 2009 (Ziemke et al., 2006).

To further investigate this validation process, monthly-mean data of OMI-MLS $\mathrm{TropoO}_{3}$ (independent data) was compared with modeled as well as observed $\mathrm{TropoO}_{3}$ concentrations over Lahore during October 2004 - September 2015 (shown in Figure 8). Monthly-mean graphical patterns, slopes, and trends in all three datasets are similar with higher concentrations during the spring and summer (monsoon) seasons, whereas, minima during the winter season shown in Figure 8(a). Comparison of scatter plots drawn between OMI-MLS $\mathrm{TropoO}_{3}$ (independent data), and observed $\mathrm{TropoO}_{3}$ (used as 
dependent variable) against modeled $\mathrm{TropoO}_{3}$ concentrations shown in Figure $8(b)$ indicates similar linear positive trends.

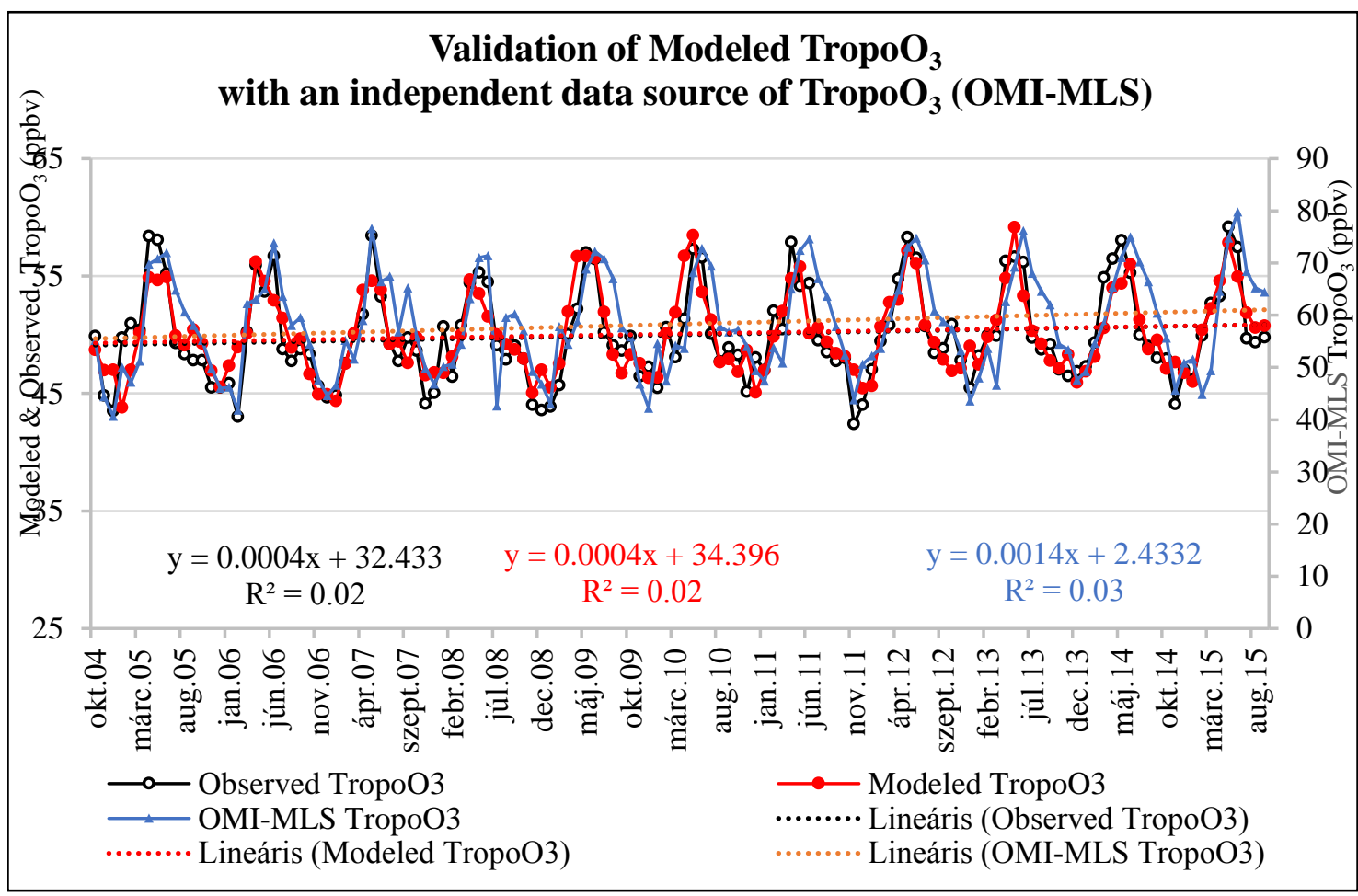

Figure 7. Validation of modeled and observed (used as dependent variable) TropoO $\mathrm{O}_{3}(\mathrm{ppbv}$ ) against an independent source of Tropo $\mathrm{O}_{3}$ concentrations ( $p p b v$ ) retrieved from OMI-MLS data product over Lahore during October 2004 - September 2015. Dotted lines correspond to respective trend lines

\section{Conclusions}

The proposed step-wise multiple linear regression model reveals weighted contributions of major factors OLR, WS, and CLF, TropoSO 2 , TropoCO, and RH (based on standardized regression coefficient, $\beta$ ) against TropoO $_{3}$ variability over Lahore during September 2004 - October 2015. Stepwise multiple linear regression modelling technique opted to model $\mathrm{TropoO}_{3}$ concentrations over Lahore has shown highly consistent results as compared with the TropoO $\mathrm{O}_{3}$ observations retrieved directly from OMI satellite sensor. The trend-line of scatter plot drawn between observed and modeled $\mathrm{TropoO}_{3}$ provides a steady linear increase analogous correlation with an overall increase of $6.18 \%$ in observed TropoO ${ }_{3}$, while $4.89 \%$ in modeled $\mathrm{TropoO}_{3}$ over Lahore during the study period. Based on the resultant value of multiple correlation coefficient $(r=0.854)$ it can confidently be asserted that the stepwise multiple linear regression model opted to predict $\mathrm{TropoO}_{3}$ over Lahore is quite reasonable. The modeled $\mathrm{TropoO}_{3}$ concentrations were validated against an independent source of $\mathrm{TropoO}_{3}$ concentrations retrieved from original subtracted product of OMI-MLS over Lahore for the study period and the results were found in good agreement. This study of modeling $\mathrm{TropoO}_{3}$ variation as functions of satellite observations of major tropospheric gases, meteorological parameters, and aerosol burden over Lahore acquired during October 2004 - September 2015 using stepwise multiple 


$$
-13549 \text { - }
$$

linear regression modelling technique is quite unique. The study would be helpful for further research investigations in this domain as well as provide useful information for policy/decision makers in Lahore, Pakistan.
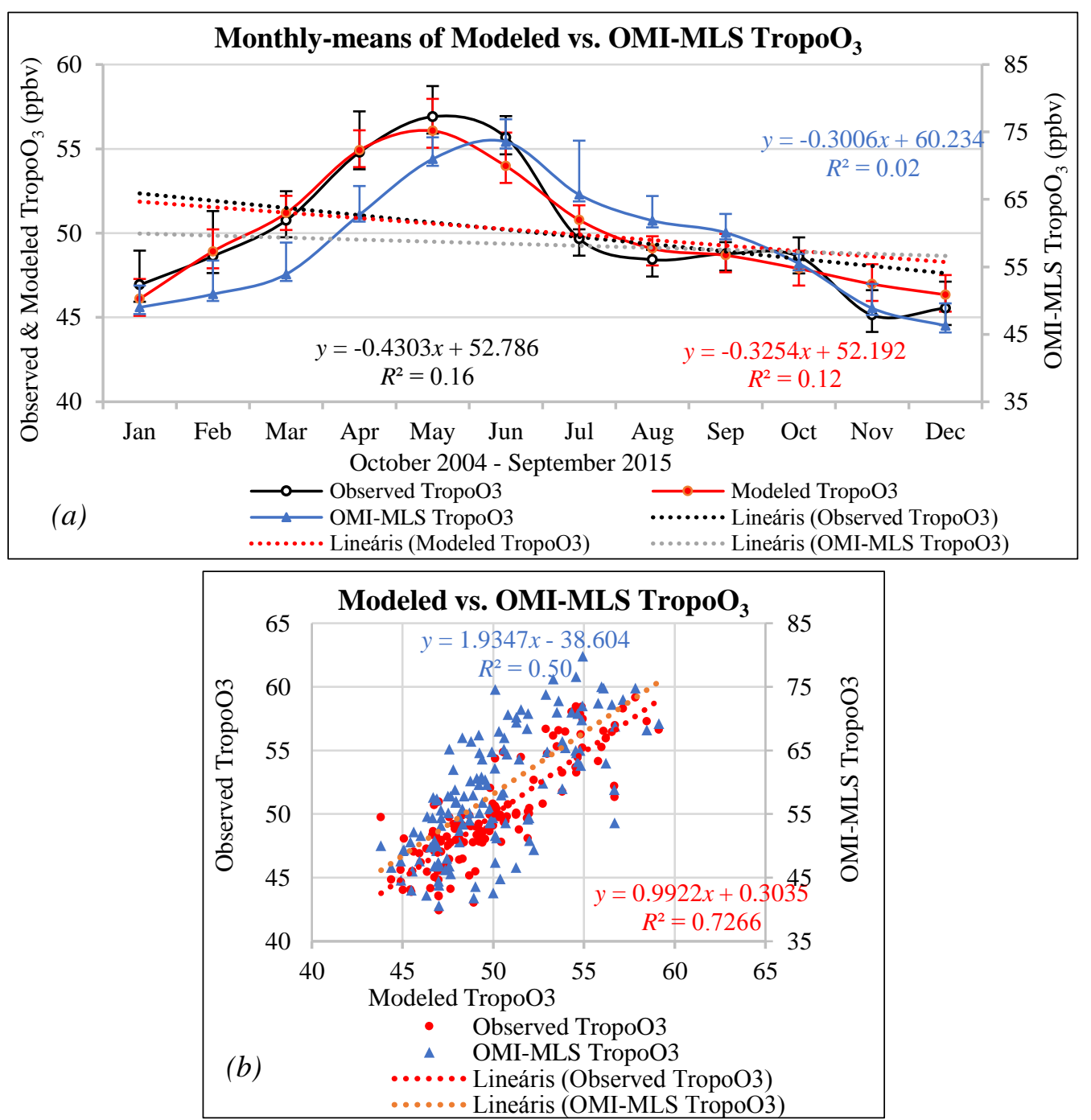

Figure 8. (a) Monthly-means of OMI-MLS TropoO 3 (independent data), observed $\mathrm{TropoO}_{3}$ (used as dependent variable) and modeled Tropo $\mathrm{O}_{3}$ concentrations (all data in ppbv) over Lahore during October 2004 - September 2015. (b) Scatter plot of OMI-MLS TropoO 3 (independent data), and observed $\mathrm{TropoO}_{3}$ (used as dependent variable) against modeled Tropo $O_{3}$ concentrations (all data in ppbv). Dotted lines correspond to respective trend lines

Acknowledgements. Authors gratefully acknowledge the project teams of AIRS/AMSU-A, GLDAS_NOAH, MODIS, OMI, and TRMM for online provision of related datasets and necessary information about error analyses.

\section{REFERENCES}

[1] Abed, F. G., Al-Salihi, A., Jasim, M. R. (2017): Space-borne observation of methane from atmospheric infrared sounder: data analysis and distribution over Iraq. - Journal of Applied and Advanced Research 2(4): 256-264. Doi:10.21839/jaar.2017.v2i4.100. 
[2] Aghedo, A. M., Schultz, M. G., Rast, S. (2007): The influence of African air pollution on regional and global tropospheric ozone. - Atmos. Chem. Phys. 7(5): 1193-1212. Doi:10.5194/acp-7-1193-2007.

[3] Ahmad, S. S., Aziz, N. (2013): Spatial and Temporal Analysis of Ground Level Ozone and Nitrogen Dioxide Concentration across the Twin Cities of Pakistan. - Environmental Monitoring and Assessment 185: 3133-3147. Doi:10.1007/s10661-012-2778-7.

[4] Alam, K., Iqbal, M. J., Blaschke, T., Qureshi, S., Khan, G. (2010): Monitoring spatiotemporal variations in aerosols and aerosol-cloud interactions over Pakistan using MODIS data. - Adv. Space Res. 46: 1162-1176. Doi:10.1016/j.asr.2010.06.025.

[5] Alam, K., Trautmann, T., Blaschke, T., Majid, H. (2012): Aerosol optical and radiative properties during summer and winter season over Lahore and Karachi. - Atmos. Environ. 50: 234-245. Doi:10.1016/j.atmosenv.2011.12.027.

[6] Alam, K., Trautmann, T., Blaschke, T., Subhan, F. (2014): Changes in aerosol optical properties due to dust storms in the Middle East and Southwest Asia. - Remote Sens. Environ. 143: 216-227. Doi:10.1016/j.rse.2013.12.021.

[7] Ali, M., Tariq, S., Mahmood, K., Daud, A., Batool, A., ul-Haq, Z. (2014): A study of aerosol properties over Lahore Pakistan) by using AERONET data. - Asia-Pacific J Atmos Sci. 50(2): 153-162. Doi: 10.1007/s13143-014-0004-y.

[8] Aumann, H. H., Chahine, M. T., Gautier, C., Goldberg, M. D., Kalnay, E., McMillin, L. M., Revercomb, H., Rosenkranz, P. W., Smith, W. L., Staelin, D. H., Strow, L. L., Susskind, J. (2003): AIRS/AMSU/HSB on the aqua mission: design, science objectives, data products, and processing systems. - IEEE Trans. Geosci. Rem. Sens. 41: 253-264. Doi: 10.1109/TGRS.2002.808356.

[9] Avnery, S., Mauzerall, D. L., Liu, J., Horowitz, L. W. (2011): Global crop yield reductions due to surface ozone exposure: 2 . Year 2030 potential crop production losses and economic damage under two scenarios of $\mathrm{O}_{3}$ pollution. - Atmos. Environ. 45(13): 2297-2309. Doi:10.1016/j.atmosenv.2011.01.002.

[10] Badarinath, K. V. S., Shailesh, K. K., Anu, R. S., Krishna, V. P. (2009): Analysis of aerosol and carbon monoxide characteristics over Arabian Sea during crop residue burning period in the Indo-Gangetic plains using multi-satellite remote sensing datasets. - J. Atmos. Sol. Terr. Phys. 71: 1267-1276. Doi:10.1016/j.jastp.2009.04.004.

[11] Bobbink, R. (1989): Impacts of tropospheric ozone and airborne nitrogenous pollutants on natural and semi-natural ecosystems: A commentary. - New Phytologist 139(1): 161-168. Doi:10.1046/j.1469-8137.1998.00175.x.

[12] Boersma, K., Eskes, H., Veefkind, J., Brinksma, E., Sneep, M., van der Oord, G., Levelt, P., Stammes, P., Gleason, J., Bucsela, E. (2006): Near-real time retrieval of tropospheric $\mathrm{NO}_{2}$ from OMI. - Atmos. Chem. Phys. Dis. 6(6): 12301-12345. Doi:10.5194/acpd-612301-2006.

[13] Burney, J., Ramanathan, V. (2014): Recent climate and air pollution impacts on Indian agriculture. - Proc. Natl. Acad. Sci. 111(46): 16319-16324.

[14] CMF (2018): City Mayors Foundation, UK. - www.citymayors.com (accessed on 11 July 2018).

[15] Cooper, O. R., Parrish, D. D., Ziemke, J., Balashov, N. V., Cupeiro, M., Galbally, I. E., Gilge, S., Horowitz, L., Jensen, N. R., Lamarque, J.-F., Naik, V., Oltmans, S. J., Schwab, J., Shindell, D. T., Thompson, A. M., Thouret, V., Wang, Y., Zbinden, R. M. (2014): Global distribution and trends of tropospheric ozone: An observation-based review. - Elem Sci Anth 2: 29. Doi:10.12952/journal.elementa.000029.

[16] El-Fadel, M., Zein, M., Nuwayhid, I., Jamali, D., Sadek, S. (2002): Environmental Management of Ozone in Beirut Urban Areas. - Environmental Management and Health 13: 471-494. Doi:10.1108/09566160210441780.

[17] Engel-Cox, J. A., Holloman, C. H., Coutant, B. W., Hoff, R. M. (2004): Qualitative and quantitative evaluation of MODIS satellite sensor data for regional and urban scale air quality. - Atmos. Environ. 38(16): 2495-2509. Doi:10.1016/j.atmosenv.2004.01.039. 
[18] EPA (2003): National air quality and emissions trends report, Special Studies Edition. Environmental Protection Agency, US EPA. EPA/454/R-03-005, North Carolina.

[19] Fang, H., Beaudoing, H. K., Rodell, M., Teng, W. L., Vollmer, B. E. (2009): Global Land Data Assimilation System (GLDAS) Products, Services and Application from NASA Hydrology Data and Information Services Center (HDISC). - ASPRS 2009 Annual Conference; 8-31 March 2009; Baltimore, MD US.

[20] Fioletov, V., Labow, G., Evans, R., Hare, E., Köhler, U., McElroy, C., Miyagawa, K., Redondas, A., Savastiouk, V., Shalamyansky, A. (2008): Performance of the ground-based total ozone network assessed using satellite data. - J. Geophys. Res. Atmos. 113(D14): 313. Doi:10.1029/2008JD009809.

[21] Ganesan, A. L., Chatterjee, A., Prinn, R. G., Harth, C. M., Salameh, P. K., Manning, A. J., Hall, B. D., Mühle, J., Meredith, L. K., Weiss, R. F., O’Doherty, S., Young, D. (2013): The variability of methane, nitrous oxide and sulfur hexafluoride in Northeast India. Atmospheric Chemistry and Physics 13: 10633-10644. Doi:10.5194/acp-13-10633-2013.

[22] Gauss, M., Myhre, G., Pitari, G., Prather, M. J., Isaksen, I. S. A., Berntsen, T. K., Brasseur, G. P., Dentener, F. J., Derwent, R. G., Hauglustaine, D. A., Horowitz, L. W., Jacob, D. J., Johnson, M., Law, K. S., Mickley, L. J., Müller, J. -F., Plantevin, P. -H., Pyle, J. A., Rogers, H. L., Stevenson, D. S., Sundet, J. K., van Weele, M., Wild, O. (2003): Radiative forcing in the 21 st century due to ozone changes in the troposphere and the lower stratosphere. $-\mathrm{J}$. Geophys. Res. 108(D9): 4292. Doi:10.1029/2002JD002624.

[23] Ghude, S. D., der Arj, V., Beig, G., Fadnavis, S., Polade, S. D. (2009): Satellite derived trends in $\mathrm{NO} 2$ over the major global hotspot regions during the past decade and their intercomparison. - Environ. Pollut. 157(6): 1873-1878. Doi:10.1016/j.envpol.2009.01.013.

[24] Holopainen, J. K. (2004): Multiple functions of inducible plant volatiles. - Trends Plant Sci. 9: 529-533.

[25] Hsu, J., Prather, M. J. (2009): Stratospheric variability and tropospheric ozone. - J. Geophys. Res. Atmos. 114:D6. http://dx.doi.org/10.1029/2008JD010942 retrieved from https://escholarship.org/uc/item/09q010cs.

[26] IPCC (2013): Climate Change 2013: The Physical Science Basis. - In: Stocker, T. F., Qin, D., Plattner, G. K., Tignor, M., Allen, S. K., Boschung, J., Nauels, A., Xia, Y., Bex, V., Midgley, P. M. (eds.). Contribution of Working Group I to the Fifth Assessment Report of the Intergovernmental Panel on Climate Change. Cambridge University Press, Cambridge, United Kingdom and New York, NY, USA, $1535 \mathrm{pp}$.

[27] Iqbal, M. A., Kim, K. -H., Shon, Z. -H., Sohn, J. -R., Jeon, E. -C., Kim, Y. -S., Oh, J. -M. (2014): Comparison of Ozone Pollution Levels at Various Sites in Seoul, a Megacity in Northeast Asia. - Atmospheric Research 138: 330-345. Doi:10.1016/j.atmosres.2013.12.003.

[28] Jacob, D. J. (2003): The oxidizing power of the atmosphere. - In: Potter, T. D., Colman, B. (eds.). Handbook of weather, climate and water. John Wiley \& Sons. pp 1-1020. ISBN: 978-0-471-21489-2.

[29] Jaeglé, L., Martin, R., Chance, K., Steinberger, L., Kurosu, T., Jacob, D. J., Modi, A., Yoboué, V., Sigha-Nkamdjou, L., Galy-Lacaux, C. (2004): Satellite mapping of raininduced nitric oxide emissions from soils. - J. Geophys. Res. Atmos. 109:D21. Doi:10.1016/j.atmosres.2013.12.003.

[30] Kulkarni, P. S., Ghude, S. D., Bortoli, D. (2010): Tropospheric ozone trend over three major inland Indian cities: Delhi, Hyderabad and Bangalore. - Anna. Geophys. 28(10): 1879-1885. Doi:10.5194/angeo-28-1879-2010.

[31] Lal, D., Ghude, S. D., Patil, S., Kulkarni, S. H., Jena, C., Tiwari, S., Srivastava, M. K. (2012): Tropospheric ozone and aerosol long-term trends over the Indo-Gangetic plain (IGP), India. - Atmos. Res. 116: 82-92. Doi:10.1016/j.atmosres.2012.02.014.

[32] Lerdau, M., Slobodkin, L. (2002): Trace gas emissions and species-dependent ecosystem services. - Trends Ecol. Evol. 17: 309-312. Doi:10.1016/S0169-5347(02)02535-1. 


$$
-13552 \text { - }
$$

[33] Levelt, P. F., van den Oord, G. H. J., Dobber, M. R., Malkki, A., Visser, H., de Vries, J., Stammes, P., Lundell, J. O. V., Saari, H. (2006a): The Ozone Monitoring Instrument. IEEE Transactions on Geoscience and Remote Sensing 44: 1093-1101. Doi:10.1109/TGRS.2006.872333.

[34] Levelt, P. F., Hilsenrath, E., Leppelmeier, G. W., van den Oord, G. B. J., Bhartia, P. K., Tamminen, J., de Haan, J. F., Veefkind, J. P. (2006b): Science objectives of the Ozone Monitoring Instrument. - IEEE Transactions on Geoscience and Remote Sensing 44(5): 1199-1208. Doi:10.1109/TGRS.2006.872336.

[35] Lin, M., Tao, J., Chan, C. -Y., Cao, J. -J., Zhang, Z. -S., Zhu, L. -H., Zhang, R. -J. (2012): Regression analyses between recent air quality and visibility changes in megacities at four haze regions in China. - Aerosol Air Qual. Res. 12: 1049-1061. Doi:10.4209/aaqr.2011.11.0220.

[36] Liu, S. N., Zhou, T., Wei, L. Y., Shu, Y. (2012): The spatial distribution of forest carbon sinks and sources in China. - Chin. Sci. Bull. 57: 1699-1707. Doi:10.1007/s11434-0124998-1.

[37] Mishra, A. K., Shibata, T. (2012): Synergistic analyses of optical and microphysical properties of agricultural crop residue burning aerosols over the Indo-Gangetic Basin (IGB). - Atmos. Environ. 57: 205-218. Doi:10.1016/j.atmosenv.2012.04.025.

[38] Noreen, A., Khokhar, M. F., Zeb, N., Yasmin, N., Hakeem, K. R. (2018): Spatio-temporal assessment and seasonal variation of tropospheric ozone in Pakistan during the last decade. - Environ. Sci. Pollut. Res. 25(9): 8441-8454. Doi:10.1007/s11356-017-1010-2.

[39] Pagano, T. S., Aumann, H. H., Hagan, D. E., Overoye, K. (2003): Prelaunch and in-flight radiometric calibration of the atmospheric infrared sounder (AIRS). - IEEE Trans. Geosci. Rem. Sens. 41(2): 265-273. Doi:10.1109/TGRS.2002.808324.

[40] Pal, C. (2010): Variability of Total Ozone over India and Its Adjoining Regions during 1997-2008. - Atmospheric Environment 44: 1927-1936. Doi:10.1016/j.atmosenv.2010.01.028.

[41] Pinto, D. M., Blande, J. D., Souza, S. R., Nerg, A. M., Holopainen, J. K. (2010): Plant Volatile Organic Compounds (VOCs) in Ozone (O3) Polluted Atmospheres: The Ecological Effects. - J Chem Ecol 36(1): 22-34. Doi:10.1007/s10886-009-9732-3.

[42] Pulikesi, M., Baskaralingam, P., Rayudu, V. N., Elango, D., Ramamurthi, V., Sivanesan, S. (2006): Surface ozone measurements at urban coastal site Chennai, in India. - Journal of Hazardous Materials 137: 1554-1559. Doi:10.1016/j.jhazmat.2006.04.040.

[43] Sahoo, A., Sarkar, S., Singh, R. P., Kafatos, M., Summers, M. E. (2005): Declining Trend of Total Ozone Column over the Northern Parts of India. - International Journal of Remote Sensing 26: 3433-3440. Doi:10.1080/01431160500076467.

[44] Satheesh, S. K., Moorthy, K. K. (2005): Radiative effects of natural aerosols: A review. Atmos. Environ. 39(11): 2089-2110. Doi:10.1016/j.atmosenv.2004.12.029.

[45] Seinfeld, J. H., Pandis, S. N. (2016): Atmospheric Chemistry and Physics: from air pollution to climate change. $3^{\text {rd }}$ Edt. - John Wiley \& Sons. ISBN-13: 978-1-118-94740-1.

[46] Shan, W. P., Yin, Y. Q., Zhang, J. D., Ding, Y. P. (2008): Observational Study of Surface Ozone at an Urban Site in East China. - Atmospheric Research 89: 252-261. Doi:10.1016/j.atmosres.2008.02.014.

[47] Sidhu, B. S., Beri, V. (2008): Rice residue management: farmer's perspective. - Indian Journal of Air Pollution Control VIII(I): 61-67.

[48] Stevenson, D., Dentener, F., Schultz, M., Ellingsen, K., Van Noije, T., Wild, O., Zeng, G., Amann, M., Atherton, C., Bell, N. (2006): Multimodel ensemble simulations of presentday and near-future tropospheric ozone. - J. Geophys. Res. Atmos. 111(D8): D08301. Doi:10.1029/2005JD006338.

[49] Streets, D. G., Canty, T., Carmichael, G. R., Defoy, B., Dickerson, R. R., Duncan, B. N., Edwards, D. P., Haynes, J. A., Henze, D. K., Houyoux, M. R., Jacob, D. J., Krotkov, N. A., Lamsal, L. N., Liu, Y., Lu, Z., Martin, R. V., Pfister, G. G., Pinderm, R. W., Salawitch, R. J., Wecht, K. J. (2013): Emissions Estimation from Satellite Retrievals: A Review of 
Current Capability. - Atmospheric Environment 77: 1011-1042. Doi:10.1016/j.atmosenv.2013.05.051.

[50] Susskind, J., Barnet, C. D., Blaisdell, J. M. (2003): Retrieval of atmospheric and surface parameters from AIRS/AMSU/HSB data in the presence of clouds. - IEEE Trans. Geosci. Rem. Sens. 41: 390-409. Doi: 10.1109/TGRS.2002.808236.

[51] Tan, K. C., Lim, H. S., MatJafri, M. Z. (2014): Analysis of Total Column Ozone in Peninsular Malaysia Retrieved from SCIAMACHY. - Atmospheric Pollution Research 5: 42-51. Doi:10.5094/APR.2014.006.

[52] The Royal Society (2008): Ground-level Ozone in the 21st Century: Future Trends, Impacts and Policy Implications. - The Royal Society, London. Science policy report 15/08. ISBN: 978-0-85403-713-1.

[53] Ul-Haq, Z., Tariq, S., Ali, M., Mahmood, K., Batool, S. A., Rana, A. D. (2014): A Study of Tropospheric $\mathrm{NO}_{2}$ Variability over Pakistan Using OMI Data. - Atmospheric Pollution Research 5: 709-720. Doi:10.5094/APR.2014.080.

[54] UNEP and WMO (2011): Integrated assessment of black carbon and tropospheric ozone. - United Nations Environment Programme, Nairobi.

[55] Van Dingenen, R., Dentener, F. J., Raes, F., Krol, M. C., Emberson, L., Cofala, J. (2009): The global impact of ozone on agricultural crop yields under current and future air quality legislation. - Atmos. Environ. 43(3): 604-618. Doi:10.1016/j.atmosenv.2008.10.033.

[56] Wang, L., Emmerich, J. S., Persily, A. K., Lina, C. C. (2012): Carbon Monoxide Generation, Dispersion and Exposure from Indoor Operation of Gasoline-Powered Electric Generators under Actual Weather Conditions. - Building and Environment 56: 283-290. Doi:10.1016/j.buildenv.2012.03.016.

[57] Xing, J., Wang, J., Mathur, R., Wang, S., Sarwar, G., Pleim, J., Hogrefe, C., Zhang, Y., Jiang, J., Wong, D.-C., Hao, J. (2017): Impacts of aerosol direct effects on tropospheric ozone through changes in atmospheric dynamics and photolysis rates. - Atmospheric Chemistry and Physics. Copernicus Publications, Katlenburg-Lindau, Germany 17: 98699883. Doi:10.5194/acp-17-9869-2017.

[58] Yienger, J. J., Levy, H. (1995): Empirical Model of Global Soil-Biogenic No $\chi$ Emissions. - Journal of Geophysical Research 100 (D6): 11447-11464. Doi:10.1029/95JD00370.

[59] Young, P., Archibald, A., Bowman, K., Lamarque, J. -F., Naik, V., Stevenson, D., Tilmes, S., Voulgarakis, A., Wild, O., Bergmann, D. (2013): Pre-industrial to end 21st century projections of tropospheric ozone from the Atmospheric Chemistry and Climate Model Intercomparison Project (ACCMIP). - Atmos. Chem. Phys. 13(4): 2063-2090. Doi:10.5194/acp-13-2063-2013.

[60] Yuan, J. S., Himanen, S., Holopainen, J. K., Chen, F., Stewart, C. N. Jr. (2009): Smelling global climate change: mitigation of function for plant volatile organic compounds. Trends Ecol. Evol. 24: 323-331. Doi: 10.1016/j.tree.2009.01.012.

[61] Ziemke, J., Chandra, S., Labow, G., Bhartia, P., Froidevaux, L., Witte, J. (2011): A global climatology of tropospheric and stratospheric ozone derived from Aura OMI and MLS measurements. - Atmos Chem Phys 11(17): 9237-9251. Doi:10.5194/acp-11-9237-2011.

[62] Ziemke, J., Olsen, M., Witte, J., Douglass, A., Strahan, S., Wargan, K., Liu, X., Schoeberl, M., Yang, K., Kaplan, T. (2014): Assessment and applications of NASA ozone data products derived from Aura OMI/MLS satellite measurements in context of the GMI chemical transport model. - J. Geophys. Res. Atmos. 119(9): 5671-5699. Doi:10.1002/2013JD020914. 